\title{
HIGH-REDSHIFT GALAXIES IN COLD DARK MATTER MODELS
}

\author{
David H. Weinberg, ${ }^{1}$ Lars Hernquist, ${ }^{2}$ and Neal Katz ${ }^{3}$ \\ Received 2000 May 12; accepted 2002 January 24
}

\begin{abstract}
We use hydrodynamic cosmological simulations to predict the star formation properties of high-redshift galaxies $(z=2-6)$ in five variants of the inflationary cold dark matter scenario, paying particular attention to $z=3$, the redshift of the largest "Lyman break galaxy" (LBG) samples. Because we link the star formation timescale to the local gas density, the rate at which a galaxy forms stars is governed mainly by the rate at which it accretes cooled gas from the surrounding medium. At $z=3$, star formation in most of the simulated galaxies is steady on $200 \mathrm{Myr}$ timescales, and the instantaneous star formation rate (SFR) is correlated with total stellar mass. However, there is enough scatter in this correlation that a sample selected above a given SFR threshold may contain galaxies with a fairly wide range of masses. The redshift history and global density of star formation in the simulations depend mainly on the amplitude of mass fluctuations in the underlying cosmological model. The three models whose mass fluctuation amplitudes agree with recent analyses of the Ly $\alpha$ forest also reproduce the observed luminosity function of LBGs reasonably well, although the dynamic range of the comparison is small and the theoretical and observational uncertainties are large. The models with higher and lower amplitudes appear to predict too much and too little star formation, respectively, although they are not clearly ruled out. The intermediate amplitude models predict SFR $\sim 30-40 M_{\odot}$ $\mathrm{yr}^{-1}$ for galaxies with a surface density of $\sim 1 \operatorname{arcmin}^{-2}$ per unit redshift at $z=3$. They predict much higher surface densities at lower SFR, and significant numbers of galaxies with SFR $>10 M_{\odot} \mathrm{yr}^{-1}$ at $z \geq 5$.

Subject headings: dark matter — galaxies: evolution — galaxies: formation -

large-scale structure of universe — stars: formation
\end{abstract}

\section{INTRODUCTION}

The discovery and characterization of "Lyman break" galaxies (LBGs) has opened a new window on the high-redshift universe, revealing a population of star-forming galaxies at $z>3$ whose comoving space density exceeds that of $L_{*}$ galaxies today (Steidel et al. 1996; Lowenthal et al. 1997). These galaxies can be identified by their unusual colors in deep imaging surveys because the intrinsic continuum break at $\lambda \sim 912 \AA$ and the intergalactic absorption by the Ly $\alpha$ forest at $\lambda<1216 \AA$ redshift into optical bands. Spectroscopic follow-up shows that photometry of Lyman break objects yields robust approximate redshifts. From an optical imaging survey, one can therefore construct a sample of high- $z$ galaxies limited primarily by rest-frame ultraviolet (UV) luminosity, which, in the absence of dust extinction, is itself determined mainly by the instantaneous formation rate of massive stars. Application of this approach to the Hubble Deep Field (HDF; Williams et al. 1996) and other deep imaging surveys has yielded first attempts at one of the long-standing goals of observational cosmology, determination of the star formation history of the universe (e.g., Madau et al. 1996; Madau 1997; Connolly et al. 1997; Steidel et al. 1999).

In this paper, we examine the ability of models based on inflation and cold dark matter (CDM) to account for the observed population of LBGs, using cosmological simulations that incorporate gravity, gas dynamics, and star for-

\footnotetext{
${ }^{1}$ Ohio State University, Department of Astronomy, Columbus, OH 43210;dhw@astronomy.ohio-state.edu.

${ }^{2}$ Harvard-Smithsonian Center for Astrophysics, Cambridge, MA 02138; lars@cfa.harvard.edu.

${ }^{3}$ University of Massachusetts, Department of Physics and Astronomy, Amherst, MA 91003; nsk@kaka.phast.umass.edu.
}

mation. We consider five variants of the CDM scenario: three $\Omega_{m}=1$ models, a spatially flat low-density model with a cosmological constant, and an open universe low-density model with $\Omega_{\Lambda}=0$. The spatial clustering of the high-redshift galaxies in these simulations was discussed by Katz, Hernquist, \& Weinberg (1999, hereafter KHW99); here we focus on the masses and star formation properties of these galaxies.

Numerical simulations play two overlapping but distinct roles in cosmological studies. First, they provide quantitative predictions that can be compared to observations in order to test the underlying cosmological models. Second, they provide greater understanding of the observational phenomena themselves by showing how observable structures might arise and evolve in a given cosmological scenario. In this paper we will emphasize the second of these roles, mainly because the numerical limitations of the simulations and our limited knowledge of the physics of star formation contribute uncertainties that are comparable to the differences between cosmological models. Indeed, of the five CDM models that we consider here, one of them (low density with a cosmological constant) appears decisively favored by recent observational developments that have nothing to do with high-redshift galaxies, including the direct evidence for cosmic acceleration from Type Ia supernovae and the combination of spatial flatness implied by microwave background anisotropy measurements with constraints on the age of the universe or the matter density (see, e.g., the recent reviews by Bahcall et al. 1999; Wang, Tegmark, \& Zaldarriaga 2002). The examination of different cosmologies is still a useful exercise, however, because it shows how cosmological parameters and properties of primordial mass fluctuations affect the predicted properties of the high-redshift galaxy population when other physical and numerical parameters are held fixed, and it provides 
insight into the physics that governs these predicted properties.

Hydrodynamic simulations complement the main alternative approach to the theoretical study of high-redshift galaxies, based on semianalytic models of galaxy formation (e.g., Baugh et al. 1998; Kauffman et al. 1999; Somerville, Primack, \& Faber 2001). Semianalytic models have advantages of flexibility and speed, making it relatively easy to explore parameter space and to compute results at all redshifts in a single calculation. However, as a necessary consequence of their analytic treatment, they rely on simplified descriptions of gravitational collapse and cooling of gas within dark halos. The strength of numerical simulations is their more realistic treatment of these processes. The only free parameters (apart from the physical parameters of the cosmological model being studied) are those related to the treatment of star formation and feedback. Given these parameters, simulations provide straightforward, untunable predictions. However, the simulation approach must contend with the numerical uncertainties caused by finite volume and finite resolution, and computational expense makes it a slow way to explore parameter space. Over the next few years, interactions between the numerical and semianalytic approaches should strengthen both. Here we mainly present the numerical results on their own terms, with a brief comparison to other hydrodynamic simulations and interpretations based on semianalytic models in $\S 5$.

We describe our numerical methods, treatment of star formation, and choice of cosmological model in $\S 2$. In $\S 3$ we present results for the LCDM model (low-density CDM with a cosmological constant) at $z=3$, the redshift best probed by recent LBG surveys. In $\S 4$ we broaden our scope, examining predictions of the five different CDM models for the population of star-forming galaxies from $z=6$ to $z=2$. We discuss our results and prospects for future progress in $\S 5$.

\section{SIMULATIONS}

\subsection{Numerical Parameters and Star Formation}

All of our simulations use TreeSPH (Hernquist \& Katz 1989; Katz, Weinberg, \& Hernquist 1996, hereafter KWH96), a code that combines smoothed particle hydrodynamics (SPH; see Lucy 1977; Gingold \& Monaghan 1977; Monaghan 1992) with a hierarchical tree algorithm (Barnes $\&$ Hut 1986) for computing gravitational forces. The method and illustrative cosmological applications are described in detail by KWH96, so here we just specify the simulation parameters and recap the points that are most important to the present investigation.

Each of our simulations uses $64^{3}$ dark matter and $64^{3}$ SPH particles to model a triply periodic volume $11.111 \mathrm{~h}^{-1}$ comoving Mpc on a side, where $h \equiv H_{0} /\left(100 \mathrm{~km} \mathrm{~s}^{-1}\right.$ $\left.\mathrm{Mpc}^{-1}\right)$. The simulations are evolved to $z=2$. For the three critical density $\left(\Omega_{m}=1\right)$ cosmological models, the dark matter particle mass is $2.76 \times 10^{9} M_{\odot}$ and the $\mathrm{SPH}$ particle mass is $1.45 \times 10^{8} M_{\odot}$. For the two low-density $\left(\Omega_{m}=0.4\right)$ models, the dark matter particle mass is $8.27 \times 10^{8} M_{\odot}$ and the SPH particle mass is $6.71 \times 10^{7} M_{\odot}$. Gravitational forces are softened using a cubic spline kernel with a softening length $\epsilon=5 h^{-1} \mathrm{kpc}$, equivalent to $\epsilon \approx 3.5 h^{-1} \mathrm{kpc}$ for a Plummer softening law. The gravitational softening length is held fixed in comoving units, i.e., $\epsilon=1.25 h^{-1}$ physical $\mathrm{kpc}$ at $z=3$. Particles have individual time steps that satisfy the conditions $\Delta t<0.4 \min \left[\epsilon /|\boldsymbol{v}|,(\epsilon /|\boldsymbol{a}|)^{1 / 2}\right]$, where $\boldsymbol{v}$ is the peculiar velocity and $\boldsymbol{a}$ is the acceleration. SPH particle time steps are also required to satisfy the Courant condition (see $\mathrm{KWH} 96$ ). The maximum time step for any particle is $\Delta t_{d}=$ $H_{0}^{-1} / 6000$.

Radiative cooling is computed assuming primordial composition gas with helium abundance $Y=0.24$ by mass. (Although the interstellar medium [ISM] of Lyman break galaxies has been enriched by local star formation, what matters for our purposes is the cooling rate of gas before it settles into the galaxies, for which low metallicity is appropriate.) All of the simulations incorporate a photoionizing UV background with the spectral shape and redshift history computed by Haardt \& Madau (1996) but with intensity reduced by a factor of 2 in order to approximately match the mean opacity of the $\operatorname{Ly} \alpha$ forest given our assumed baryon density (Croft et al. 1997). In practice, the photoionizing background has negligible effect on the LBG population, at least in the mass range that our simulations can resolve (Weinberg, Hernquist, \& Katz 1997a).

The gas that resides in collapsed dark matter halos exhibits a two-phase structure: hot gas at roughly the halo virial temperature with a density profile similar to that of the dark matter (but exhibiting a core at small radii), and radiatively cooled gas with $T \sim 10^{4} \mathrm{~K}$ at much higher overdensity. In simulations that do not incorporate star formation, the clumps of radiatively cooled gas have masses and sizes comparable to the luminous regions of observed galaxies. Our star formation algorithm is essentially a prescription for turning this dense, cold gas into collisionless stars, returning energy from supernova feedback to the surrounding medium. We provide a brief synopsis of this algorithm here and refer the reader to KWH96 for details.

An SPH gas particle is "eligible" to form stars if it is Jeans unstable, resides in a region of converging flow, has an overdensity $\rho_{g} / \overline{\rho_{g}}>55.7$ (corresponding to the virial boundary of a singular isothermal sphere in the spherical collapse model), and has a hydrogen number density exceeding $0.1 \mathrm{~cm}^{-3}$ (physical units). In practice, it is the physical density threshold that matters - gas with this density almost always satisfies the other criteria, except at very high redshift, where the overdensity threshold ensures that star formation does not occur in uncollapsed regions simply because the cosmic mean density is high. Once a gas particle is eligible to form stars, its star formation rate is given by

$$
\frac{d \rho_{*}}{d t}=-\frac{d \rho_{g}}{d t}=\frac{c_{*} \epsilon_{*} \rho_{g}}{t_{g}},
$$

or

$$
\frac{d \ln \rho_{g}}{d t}=-\frac{c_{*} \epsilon_{*}}{t_{g}},
$$

where $c_{*}$ is a dimensionless star formation rate (SFR) parameter, $\epsilon_{*}$ is the fraction of the particle's gas mass that will be converted to stellar mass in a single simulation time step, and the gas flow timescale $t_{g}$ is the maximum of the local gas dynamical time, $t_{\mathrm{dyn}}=\left(4 \pi G \rho_{g}\right)^{-1 / 2}$, and the local cooling time. Each SPH particle has both a gas mass and a stellar mass (initially zero); the total gas+stellar mass contributes to gravitational forces, but only the gas mass is used in computing the SPH properties and forces. The probability $p$ that an eligible SPH particle undergoes a star forma- 
tion event in an integration time step of duration $\Delta t$ is

$$
p=1-\exp \left(\frac{-c_{*} \Delta t}{t_{g}}\right),
$$

and if the particle does undergo such an event, then $\epsilon_{*}=\frac{1}{3}$ of its remaining gas mass is converted into stars during that step. In the limit (nearly always satisfied in the simulations) that $c_{*} \Delta t / t_{g} \ll 1$, this algorithm yields the average star formation rate given by equation (1). ${ }^{4}$ Once a particle's gas mass falls below $5 \%$ of its original mass, it is converted into a collisionless, pure star particle, affected only by gravity, and its residual gas mass is redistributed to its SPH neighbors.

When an SPH particle undergoes star formation, recycled gas and supernova feedback energy are distributed to the particle and its neighbors, assuming a Miller-Scalo (Miller $\&$ Scalo1979) initial mass function (IMF) truncated at 0.1 and $100 M_{\odot}$ and $10^{51}$ ergs supernova ${ }^{-1}$. This feedback energy is usually radiated away because it is released into a dense, gas-rich medium with a short cooling time. Therefore, feedback has only a modest impact in our simulations, and this is the physically appropriate result if the protogalactic interstellar medium is fairly smooth and as dense as our simulations imply. It is possible that strong inhomogeneities in the ISM (on scales well below our resolution limits) allow feedback to have a stronger effect in real protogalaxies, and explicit modeling of this possibility is an important direction for future investigation. The scenario that we investigate here is a physically plausible limiting case.

In all of our simulations we set the SFR parameter $c_{*}$ to 0.1 and $\epsilon_{*}$ to $\frac{1}{3}$. As shown in KWH96, the stellar masses of the simulated galaxies are insensitive to the value of $c_{*}$. In the KWH96 tests, an order-of-magnitude increase to $c_{*}=1.0$ changes the total stellar mass in the box at $z=2$ by only $15 \%$, and the effect of a higher $c_{*}$ is actually to decrease the stellar mass because star formation occurs in lower density gas where supernova feedback can have a stronger effect. Indeed, one obtains nearly the same galaxy population in simulations that do not include star formation at all, except that in this case the "galaxies" are the clumps of cold, dense gas instead of the clumps of cold, dense gas and stars (see KWH96, Fig. 5). In our simulations, the rate at which a galaxy forms stars is governed mainly by the rate at which gas condenses from the hot halo into the cold clump; the regulation implied by equation (1) ensures that the gas condensation rate and the star formation rate cannot get too far out of step. The link between star formation rate and gas density is physically motivated, since denser gas is more gravitationally unstable and more easily able to radiate its energy. In the case where the cooling timescale is short and $t_{g}=t_{\text {dyn }}$, equation (1) implies $\dot{\rho}_{*} \propto \rho_{g}^{3 / 2}$, similar to the Schmidt law $\dot{\rho}_{*} \propto \Sigma_{g}^{3 / 2}$ observed to hold over a large dynamic range in a wide variety of local galaxies (Schmidt 1959; Kennicutt 1998).

\subsection{Cosmological Models}

We consider five different cosmological models, all of which assume Gaussian primordial fluctuations and a uni-

\footnotetext{
${ }^{4}$ In KWH96, the description of the algorithm is accurate but their eqs. (44) and (45), which correspond to eqs. (1) and (2), are missing the factor of $\epsilon_{*}$.
}

verse dominated by cold, collisionless dark matter. In all cases we adopt a baryon density parameter $\Omega_{b}=0.0125 h^{-2}$ based on Walker et al. (1991), although a higher $\Omega_{b}$ is suggested by recent analyses of the Ly $\alpha$ forest opacity (Rauch et al. 1997; Weinberg et al. 1997b) and the deuterium abundance in high-redshift Lyman limit systems (Burles \& Tytler 1997, 1998). The model we refer to as "standard" CDM (SCDM) assumes $\Omega_{m}=1, h=0.5$, and an rms linear theory fluctuation in $8 h^{-1} \mathrm{Mpc}$ spheres of $\sigma_{8}=0.7$. For this model we use the parameterization of the CDM power spectrum given by Bardeen et al. (1986). The $\sigma_{8}=0.7$ normalization is roughly consistent with the observed abundance of rich galaxy clusters (White, Efstathiou, \& Frenk 1993), but the SCDM model does not reproduce the amplitude of cosmic microwave background anisotropies observed by the COBE Differential Microwave Radiometer (DMR) experiment (Smoot et al. 1992; Bennett et al. 1996). Our second model, $C O B E$-normalized CDM (CCDM), is the same as SCDM except that the normalization $\sigma_{8}=1.2$ is chosen to match the 4 yr $C O B E$ data (Gorski et al. 1996; see Bunn \& White 1997 for a discussion of the CDM normalization). With this value of $\sigma_{8}$ and $\Omega_{m}=1$, the CCDM model produces galaxy clusters that are too massive to be consistent with observations.

One way to reconcile the $C O B E \mathrm{DMR}$ anisotropies and the observed cluster abundance within the context of $\Omega_{m}=1$ CDM models is to assume that inflation generates a primeval power spectrum that is "tilted" instead of scaleinvariant, $P(k) \propto k^{n}$ with $n<1$. For our tilted CDM (TCDM) model, we adopt $n=0.80$ and the transfer function given by equation (D28) of $\mathrm{Hu} \&$ Sugiyama (1996), which treats baryon damping effects more accurately than the original Bardeen et al. (1986) formulation. We normalize this analytic fit to the power spectrum to the amplitude $\sigma_{8}=0.54$ implied by $C O B E$ normalization, which we compute using the CMBFAST code of Seljak \& Zaldarriaga (1996; Zaldarriaga, Seljak, \& Bertschinger 1998), assuming the standard tensor mode contribution to microwave background anisotropies predicted by power-law inflation models.

Another way to resolve the $C O B E$ /cluster conflict is to lower the value of $\Omega_{m}$, reducing cluster masses for a given $\sigma_{8}$. We consider two different low $-\Omega_{m}$ CDM models, one with a spatially flat universe (LCDM) and a cosmological constant $\Omega_{\Lambda}=1-\Omega_{m}$ and one with an open universe $(\mathrm{OCDM})$ and $\Omega_{\Lambda}=0$. For LCDM we adopt $\Omega_{m}=0.4$, $h=0.65$, and a primeval spectral index $n=0.93$. With the tensor mode contribution, CMBFAST implies a normalization $\sigma_{8}=0.8$, which provides a good match to the cluster abundances for $\Omega_{m}=0.4$ (White et al. 1993). We again use the $\mathrm{Hu} \&$ Sugiyama (1996) formulation of the transfer function. For OCDM, we adopt $\Omega_{m}=0.4, h=0.65, n=1.0$, and a 2 yr $C O B E$ DMR normalization $\sigma_{8}=0.75$ (Ratra et al. 1997). Cluster masses in this model are lower than those in TCDM or LCDM, but they are consistent with current observations given their uncertainties (Cole et al. 1997). For OCDM we use the transfer function of Efstathiou, Bond, \& White (1992) with $\Gamma=0.234$; the $\mathrm{Hu} \&$ Sugiyama (1996) formulation is more accurate, but we were unaware of it at the time we ran the OCDM simulation. In practice, the differences between different analytic or numerical formulations of transfer functions are of the same magnitude as the changes caused by slight shifts in the adopted values of $h$, $\Omega_{b}$, or $\Omega_{m}$. 
TABLE 1

Cosmological Model Parameters

\begin{tabular}{ccccccccc}
\hline \hline Model & $\Omega_{m}$ & $\Omega_{\Lambda}$ & $h$ & $\Omega_{b}$ & $\sigma_{8}$ & $n$ & $\begin{array}{c}60 m_{\text {dark }} \\
\left(M_{\odot}\right)\end{array}$ & $\begin{array}{c}60 m_{\text {SPH }} \\
\left(M_{\odot}\right.\end{array}$ \\
\hline CCDM ...... & 1.0 & 0.0 & 0.50 & 0.05 & 1.2 & 1.0 & $1.7 \times 10^{11}$ & $8.7 \times 10^{9}$ \\
SCDM ....... & 1.0 & 0.0 & 0.50 & 0.05 & 0.7 & 1.0 & $1.7 \times 10^{11}$ & $8.7 \times 10^{9}$ \\
OCDM ...... & 0.4 & 0.0 & 0.65 & 0.03 & 0.75 & 1.0 & $5.0 \times 10^{10}$ & $4.0 \times 10^{9}$ \\
LCDM ....... & 0.4 & 0.6 & 0.65 & 0.03 & 0.8 & 0.93 & $5.0 \times 10^{10}$ & $4.0 \times 10^{9}$ \\
TCDM ....... & 1.0 & 0.0 & 0.50 & 0.05 & 0.54 & 0.80 & $1.7 \times 10^{11}$ & $8.7 \times 10^{9}$
\end{tabular}

Parameters of the five cosmological models are listed in Table 1. Figure 1 shows the linear theory power spectra of the five models, over the range of scales represented in the initial conditions of our simulations. Instead of $P(k)$ itself, we plot $\Delta^{2}(k) \equiv 4 \pi k^{3} P(k)$, which (with our Fourier transform convention) is the contribution to the variance of linear mass fluctuations per unit interval of $\ln k$ (see Peacock \& Dodds 1994). The differences in fluctuation amplitude among the three $\Omega_{m}=1$ models are easy to see, as is the difference in $P(k)$ shape between the CCDM/SCDM models and the TCDM and OCDM/LCDM models. Although LCDM has a slightly higher normalization than OCDM at $z=0\left(\sigma_{8}=0.80\right.$ vs. $\left.\sigma_{8}=0.75\right)$, OCDM has higher amplitude fluctuations at $z=3$ because of the smaller ratio of linear growth factors between $z=3$ and $z=0$ in an open universe. As already noted in the introduction, LCDM is clearly the model most favored by current observational data, but the comparison between models allows us to study the influence of cosmological parameters on the predicted properties of LBGs and to better understand the physics that determines these properties.

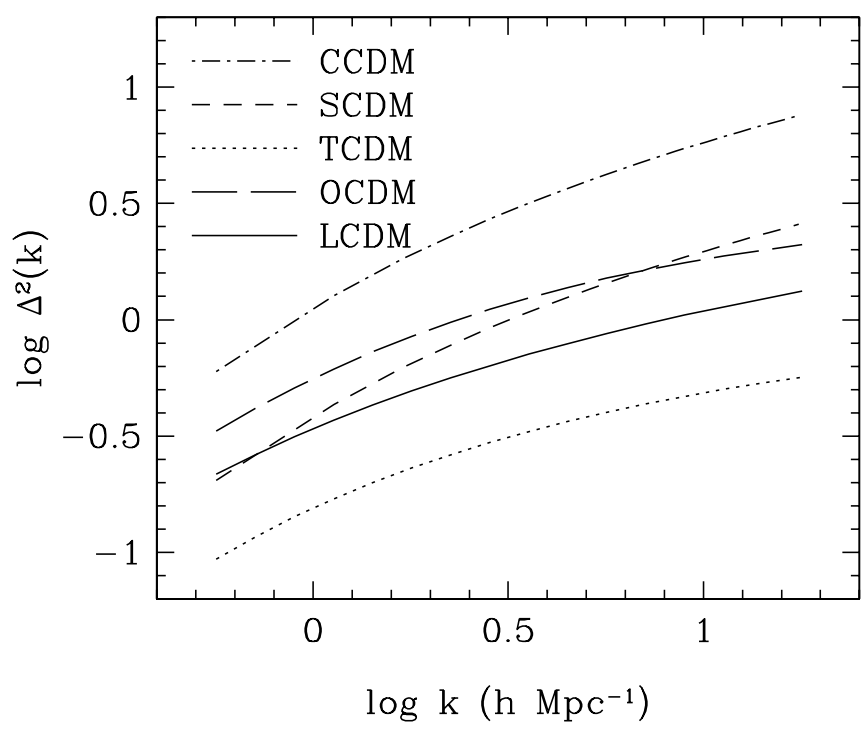

FIG. 1.-Amplitude of linear mass fluctuations in the different cosmological models at $z=3$. The quantity $\Delta^{2}(k) \equiv 4 \pi k^{3} P(k)$ is the contribution to the variance of fluctuations per unit interval of $\ln k$. The wavenumber $k$ is in comoving $h \mathrm{Mpc}^{-1}$. The range shown is from the fundamental mode of the simulation cube, $k_{f}=2 \pi /\left(11.111 h^{-1} \mathrm{Mpc}\right)$, to the Nyquist frequency of the initial particle grid, $k_{\mathrm{N}}=32 k_{f}$.

\section{LYMAN BREAK GALAXIES IN THE LCDM MODEL}

We will focus in this section on the galaxy population of the LCDM model at $z=3$, then turn to other models and other redshifts in $\S 4$. Figure 2 shows particle distributions at $z=3$ from the full $11.111 h^{-1} \mathrm{Mpc}$ simulation cube (top panels) and a $0.09 h^{-1} \mathrm{Mpc}$ subcube containing the richest concentration of galaxies (bottom panels). The top panels show numerous concentrations of dense, shock-heated gas, with typical temperatures $T \sim 10^{6} \mathrm{~K}$ corresponding to the virial temperatures of the corresponding dark matter halos. However, the zoomed view at the lower left reveals the twophase structure that characterizes collapsed regions of the simulation. Extremely overdense clumps of $10^{4} \mathrm{~K}$ gas, typically a few kpc in size, reside in a background of gas with $T \gtrsim 10^{6} \mathrm{~K}$. These dense concentrations of cold gas are, of course, the sites of star formation, as shown in the lower right panel. Because the knots of cold gas and stars stand out so clearly from the background, there is no ambiguity in identifying the galaxies in such a simulation; one only needs an algorithm that picks out these clumps. We use the SKID algorithm (Spline-Kernel Interpolated DENMAX; see KWH96 and Gelb \& Bertschinger 1994), as implemented by Stadel, Katz, \& Quinn, ${ }^{5}$ which identifies clumps of gravitationally bound particles associated with a common density maximum.

In collapsing dark matter halos that contain a small number of particles, the resolution of the SPH calculation becomes too low for the simulation to follow the cooling of the gas and subsequent star formation. Gardner et al. (1997, 2001a) find that simulated halos with at least 60 dark matter particles nearly always contain a cold gas concentration, while halos with fewer particles often do not. We can therefore resolve the existence of galaxies in halos with mass $M>60 m_{\text {dark }}$, a quantity that we list in Table 1 as an indication of our dark matter mass resolution. At $z=3$, the halo circular velocity corresponding to this limiting mass (plus the associated baryon mass) is $v_{c} \sim 140 \mathrm{~km} \mathrm{~s}^{-1}$ in the $\Omega_{m}=1$ models and $v_{c} \sim 95 \mathrm{~km} \mathrm{~s}^{-1}$ in the low-density models (see Gardner et al. 1997,eq. [3]). Comparison of LCDM simulations with $64^{3}$ and $128^{3}$ SPH particles in the same $11.111 h^{-1} \mathrm{Mpc}$ volume suggests that the requirement for accurate estimation of a galaxy's star formation rate via equation (1) is somewhat more stringent, corresponding to 60 or more particles in the condensed baryon phase (cold gas plus stars); in lower mass systems, gas densities and, therefore, star formation rates are systematically underestimated. We list $60 m_{\mathrm{SPH}}$ as an approximate baryon mass resolution limit for star formation calculations in Table 1. (The $128^{3}$ simulation is described by Gardner et al. 2001a. We will carry out a detailed investigation of resolution and box size effects on simulated galaxy populations in a future paper, using a larger suite of LCDM simulations.)

For the galaxies at $z=3$, the asterisks in Figure 3 compare the star formation rate averaged over the previous 20 Myr to the star formation rate averaged over the previous $200 \mathrm{Myr}$. The two rates are usually within a factor of 2 of each other and are often much closer, indicating that star formation in our simulated galaxies is fairly steady over intervals of 200 Myr. Circles in Figure 3 show the "instantaneous" star formation rate calculated by applying the pre-

\footnotetext{
${ }^{5}$ See http://www-hpcc.astro.washington.edu/tools/SKID/.
} 

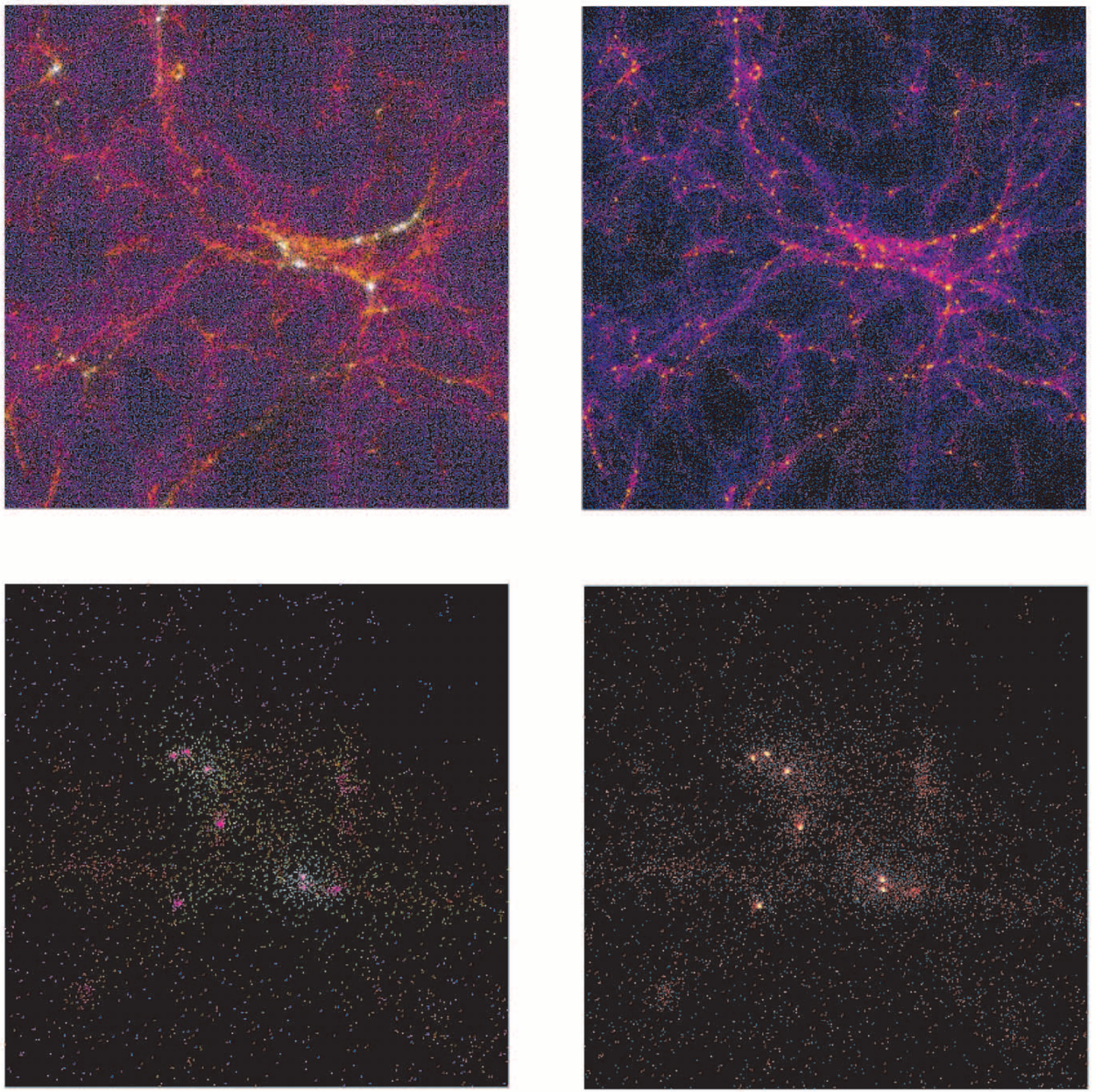

FIG. 2.-Gas and galaxies in the LCDM simulation at $z=3$. Upper panels show the gas distribution in the full $11.111 h^{-1}$ Mpc simulation cube (comoving size), color-coded by $\log T$ (left) and by $\log \rho_{g}$ (right). The lower left panel shows the gas temperature in a cubical subvolume $0.09 h^{-1} \mathrm{Mpc}(\mathrm{comoving})$ on a side. The lower right panel shows the dark matter (blue), gas (red) and star (yellow) particles in the same subvolume. The temperature scale in the left hand panels runs from several thousand Kelvin (blue) to $10^{4} \mathrm{~K}$ (magenta), $10^{5} \mathrm{~K}$ (orange/yellow), and $\geq 10^{6} \mathrm{~K}$ (white).

scription of $\S 2$ to the gas particle distribution. This is the rate that would be used to calculate star formation in the simulation's next system time step (of duration $\Delta t=2.5$ Myr). The tight correlation between this estimate of the star formation rate and the rates averaged over longer intervals demonstrates that the instantaneous estimate is not sensitive to numerical " noise" in particle densities and positions, at least for systems with $\mathrm{SFR}>1 M_{\odot} \mathrm{yr}^{-1}$. We will henceforth use the instantaneous estimate as our standard measure of the SFR, with Figure 3 as evidence that our results are insensitive to this choice.

Figure 4 plots galaxies' instantaneous star formation rates against their stellar masses. The correlation between
SFR and $M_{*}$ is reasonably strong, but there is enough scatter that a sample selected by a threshold in SFR (above a horizontal line in Fig. 4) excludes some fairly massive galaxies and includes others that are substantially farther down the stellar mass function. Nearly all galaxies are forming stars at a rate faster than the rate $\langle\mathrm{SFR}\rangle=M_{*} / t$ that would build them up steadily over the age of the universe; this result is not surprising, since the galaxies do not start to form stars at $t=0$. The ratio $\mathrm{SFR} /\langle\mathrm{SFR}\rangle$ is substantially higher for low-mass galaxies than for high-mass galaxies, probably because they have not yet used up as much of their gas supply (see below). Since this ratio is correlated with the overall shape of a galaxy's spectral energy distribution, Fig- 


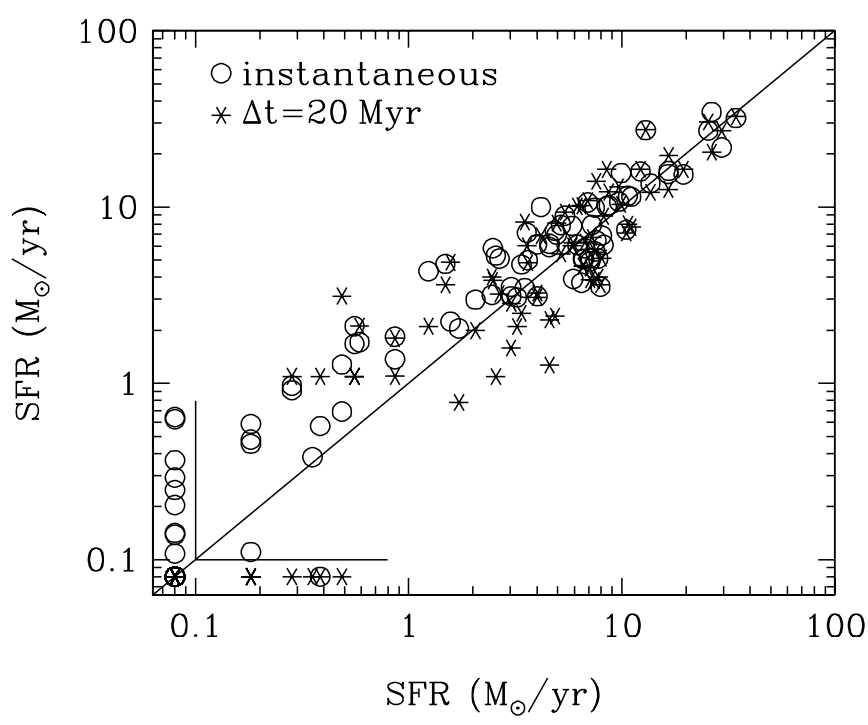

FIG. 3.-Dependence of SFR on time-averaging, for simulated galaxies in the LCDM model at $z=3$. The $x$-axis is the galaxy SFR averaged over $\Delta t=200$ Myr. Open circles show the instantaneous SFR, estimated by applying the prescription of $\S 2$ (see eq. [1]) to the simulated gas distribution. Asterisks show the SFR averaged over $\Delta t=20 \mathrm{Myr}$. Points along the axis have SFR $<0.1 M_{\odot} \mathrm{yr}^{-1}$. The age of the universe at this redshift is 1.97 Gyr.

ure 4 implies that less massive galaxies should be bluer than more massive galaxies, once the spectral energy distributions are corrected for internal reddening by dust. This trend could be caused partly by our limited numerical resolution, since the simulated galaxies do not form stars at the correct efficiency until they are sufficiently massive. However, the trend appears to be present even in the fairly wellresolved systems.

Figure 5 plots the ratio of stellar mass to baryon mass (stars plus cold gas) as a function of baryon mass. The low-

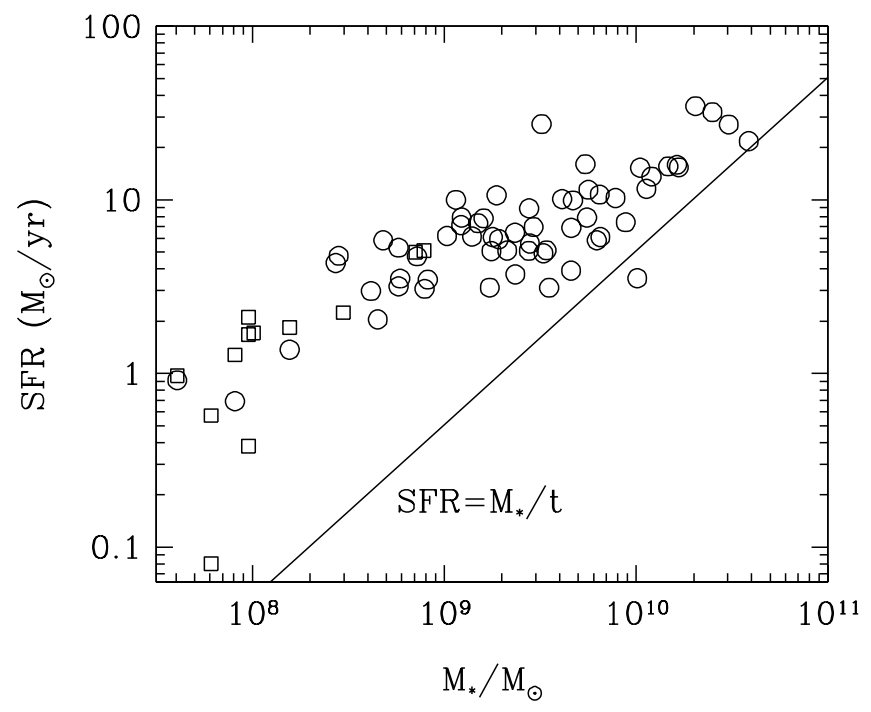

FIG. 4.-Correlation between instantaneous SFR and stellar mass, for simulated galaxies in the LCDM model at $z=3$. The solid line shows the relation SFR $=M_{*} / t$ that would apply for galaxies forming stars at a constant rate over the age of the universe, $t=1.97 \mathrm{Gyr}$. Squares represent galaxies with baryonic mass $M<60 m_{\mathrm{SPH}}$, for which the SFR is likely to be underestimated because of limited numerical resolution.

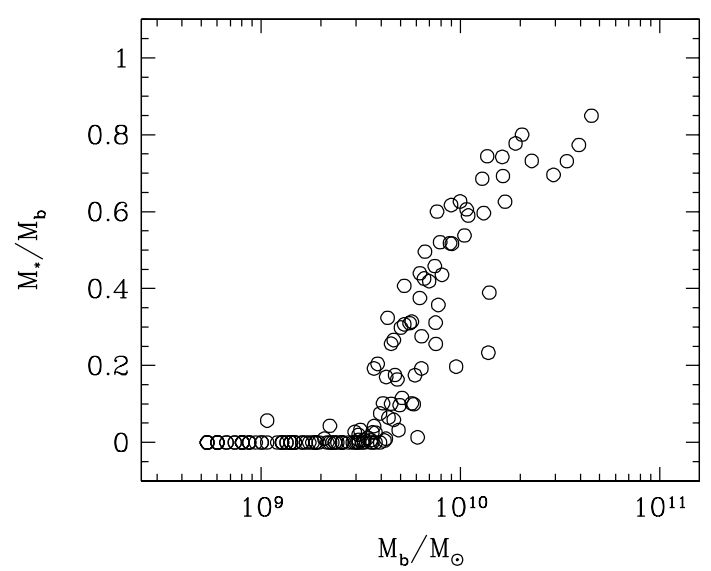

FIG. 5.- Stellar mass fraction as a function of total baryonic mass for simulated galaxies in the LCDM model at $z=3 . M_{*}$ is the stellar mass, and $M_{b}$ is the total mass of stars and cold dense gas. Our estimated minimum mass for correct calculation of the SFR is $M_{b}=60 m_{\mathrm{SPH}}=4 \times 10^{9} M_{\odot}$. However, the trend of stellar mass fraction with total mass may be exaggerated by numerical resolution effects even above this threshold, since galaxies near the threshold may have had lower masses, and underestimated SFRs, at earlier times.

mass galaxies are gas-rich, while the most massive systems are predominantly stellar. This trend is physically plausible, but it is almost certainly exaggerated by our underestimate of star formation rates in poorly resolved systems. It should therefore be treated as a tentative prediction, awaiting confirmation with higher resolution simulations.

All of the simulated galaxies reside in dark matter halos, and the more massive halos frequently contain several galaxies (see Gardner et al. 2001a, Figs. 6 and 7). As a characteristic of these halos, we calculate the circular velocity $v_{c}=(G M / r)^{1 / 2}$ from the total mass (dark plus baryonic) within a physical radius $r=40 h^{-1} \mathrm{kpc}$ around each simulated galaxy. Figure 6 plots these circular velocities against the baryon masses (left) and star formation rates (right) of the LCDM galaxies at $z=3$. There is a well-defined lower ridge line in the $v_{c}$ versus $M_{b}$ plot, but there are also galaxies with low $M_{b}$ and high $v_{c}$, which are usually "satellite" galaxies in halos with several distinct baryonic subunits. The galaxies with high SFR tend to reside in relatively massive halos, but halos above a given $v_{c}$ threshold host galaxies with a wide range of SFR, and the correlation between SFR and halo $v_{c}$ becomes increasingly weak below SFR $=10 M_{\odot}$ $\mathrm{yr}^{-1}$. Changing the radius for $v_{c}$ definition from $r=40 h^{-1} \mathrm{kpc}$ to $r=20 h^{-1} \mathrm{kpc}$ or $r=80 h^{-1} \mathrm{kpc}$ makes little difference to the appearance of Figure 6. For the galaxies with high SFR, the circular velocities in Figure 6 are large compared to the typical nebular emission line widths $\sigma \approx 70 \mathrm{~km} \mathrm{~s}^{-1}$ measured in LBGs (Pettini et al. 1998, 2001), but it is unclear that emission line widths have much to do with the dark matter potential well depth even in local starburst galaxies (Lehnert \& Heckman 1996).

Figure 7 illustrates the build-up of the galaxy population in the LCDM model from $z=6$ to $z=2$. Each panel shows a projection of the box at the indicated redshift, with each galaxy represented by a circle whose area is proportional to the instantaneous star formation rate. By $z=6$ there are already 10 galaxies in the box with SFR $>1 M_{\odot} \mathrm{yr}^{-1}$ and two with SFR $>10 M_{\odot} \mathrm{yr}^{-1}$. As time goes on, the star formation rates of the most massive galaxies tends to increase, although this trend saturates after $z=4$. The total number 

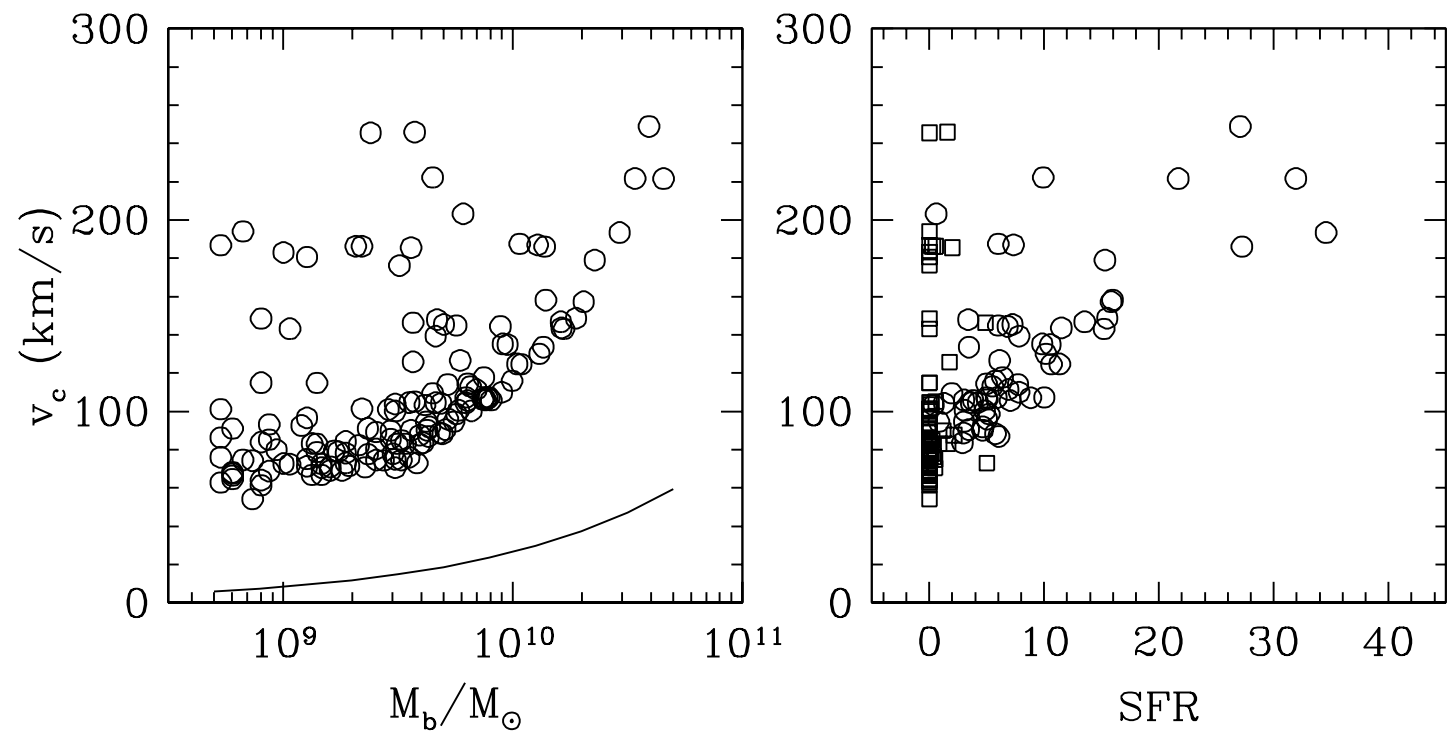

FIG. 6.-Circular velocity $v_{c}=(G M / r)^{1 / 2}$ computed based on the total mass within $r=40 h^{-1} \mathrm{kpc}$ (physical) around simulated galaxies in the LCDM model at $z=3$, as a function of galaxy baryon mass (left) and instantaneous SFR (right). The solid line in the left panel shows the contribution to the circular velocity from condensed baryons alone, $v_{c}=\left(G M_{b} / r\right)^{1 / 2}$, again for $r=40 h^{-1} \mathrm{kpc}$. In the right panel, squares represent galaxies with $M_{b}<60 m_{\mathrm{SPH}}=4 \times 10^{9} M_{\odot}$.

of star-forming galaxies in the box increases steadily, with more than 200 galaxies in the box at $z=2$. Most strikingly, the locations of newly forming galaxies are strongly correlated with the locations of preexisting galaxies so that the backbone of structure traced by the galaxy population remains similar from $z=6$ to $z=2$, although it becomes better defined as more galaxies form. We show in KHW99 that the galaxy correlation function $\xi(r)$ remains nearly constant in comoving coordinates from $z=4$ to $z=2$ and displays little dependence on the cosmological model, although the amplitude of $\xi(r)$ does increase at a given redshift if one selects only the most massive systems. Comparisons of other models in forms similar to Figure 7 appear in KHW99 (see their Fig. 1).

\section{HIGH-REDSHIFT STAR FORMATION IN CDM COSMOLOGIES}

We now turn to the main quantitative results of the paper, predictions of the star formation rates of high-redshift galaxies in our simulations of the five CDM models listed in Table 1. Each simulation represents a theoretical model that combines cosmological assumptions with assumptions about galactic scale star formation. While the predictions are not sensitive to the value of $c_{*}$, the one free parameter in our star formation algorithm, they do depend on the general features of the algorithm itself: the star formation rate is an increasing function of the local density of cold gas, and supernova feedback energy is deposited mainly in the dense interstellar medium of forming galaxies and is therefore radiated away rather efficiently. We return to this point in $\S 5$.

Figure 8 shows the cumulative distribution of the simulated galaxies as a function of instantaneous SFR, at redshifts $z=6,5,4,3$, and 2 . The vertical axis represents the comoving space density of all simulated galaxies with star formation rate exceeding the indicated SFR, in $h^{3} \mathrm{Mpc}^{-3}$. The amplitudes and redshift evolution of these distributions depend strongly on the amplitude of mass fluctuations in the cosmological model (see Fig. 1). The CCDM simulation, with the highest fluctuation amplitude, already has 15 galaxies with SFR $>60 M_{\odot} \mathrm{yr}^{-1}$ by $z=6$. The number of rapidly star-forming galaxies declines slowly from $z=5$ to $z=3$ and drops substantially between $z=3$ and $z=2$, although even at this redshift the CCDM model has the highest overall star formation rate of any of the models. The TCDM model, with the lowest fluctuation amplitude, has no star formation in galaxies resolved by our simulation until $z=4$. It exhibits a rapid rise in the number of starforming galaxies between $z=4$ and $z=3$, and a steepening of the distribution function from $z=3$ to $z=2$. The flatness of the $z=3$ distribution function may be in part a numerical artifact, since the lower mass systems in this low-amplitude model are barely resolved, and their star formation rates may be correspondingly underestimated. The other three models, with intermediate fluctuation amplitude, show intermediate behavior. For example, the LCDM model displays a steady rise in the star formation rate from $z=6$ to $z=4$, then little change from $z=4$ to $z=2$.

To facilitate comparison between Figure 8 and existing or future observational data, we list in Table 2 the conversions from SFR to apparent magnitude and from comoving $h^{3} \mathrm{Mpc}^{-3}$ to number $\operatorname{arcmin}^{-2}$ per unit redshift. Specifically, $C_{V}$ is the volume conversion factor, and $m_{10}$ is the apparent magnitude on the $\mathrm{AB}$ system at observed wavelength $\lambda_{\text {obs }}=1500 \times(1+z) \AA$ that corresponds to $\mathrm{SFR}=10 M_{\odot} \mathrm{yr}^{-1}$. The value of $m_{10}$ is similar in our highand low-density models because the effect of low- $\Omega_{m}$ is approximately canceled by the increase in $H_{0}$. We adopt the conversion from SFR to UV continuum luminosity quoted by Pettini et al. (1998), $L_{1500}=10^{29}\left(\mathrm{SFR} / 10 M_{\odot} \mathrm{yr}^{-1}\right)$ ergs s${ }^{-1} \mathrm{~Hz}^{-1}$, which in turn is based on Bruzual \& Charlot (1993) population synthesis models assuming continuous star formation and a Salpeter IMF extending from 0.1 to $100 M_{\odot}$. For example, the LCDM model predicts $10^{-2.66}$ galaxies per $h^{-3} \mathrm{Mpc}^{3}$ with SFR above $20 M_{\odot} \mathrm{yr}^{-1}$ at $z=5$, corresponding to $C_{V} n=664 \times 10^{-2.66}=1.46$ galaxies $\operatorname{arcmin}^{-2}$ per unit redshift with apparent $\mathrm{AB}$ magni- 


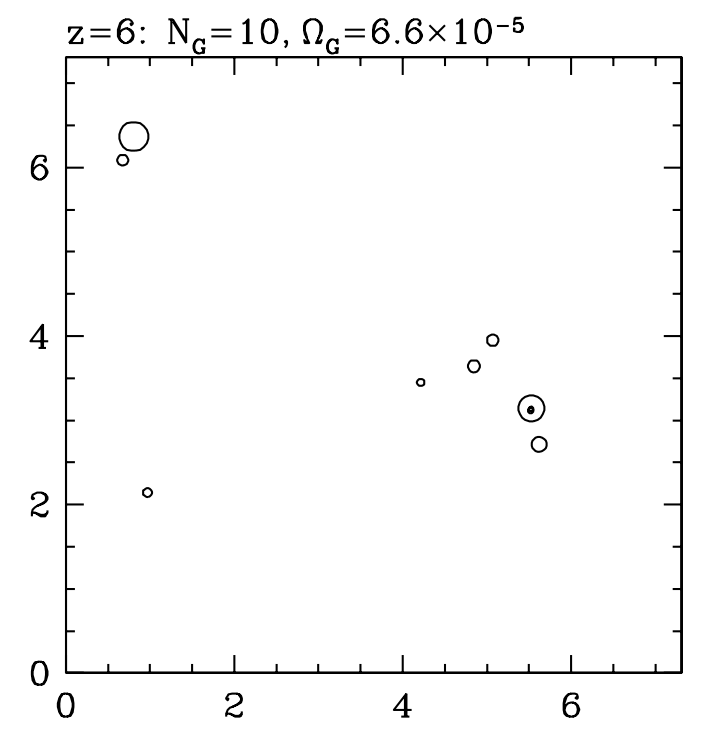

$$
\begin{array}{ll}
\bigcirc & 40 \mathrm{M}_{\odot} / \mathrm{yr} \\
\bigcirc & 20 \mathrm{M}_{\odot} / \mathrm{yr} \\
0 & 10 \mathrm{M}_{\odot} / \mathrm{yr} \\
\circ & 5 \mathrm{M}_{\odot} / \mathrm{yr} \\
\circ & 2 \mathrm{M}_{\odot} / \mathrm{yr} \\
\text { - } 1 \mathrm{M}_{\odot} / \mathrm{yr}
\end{array}
$$
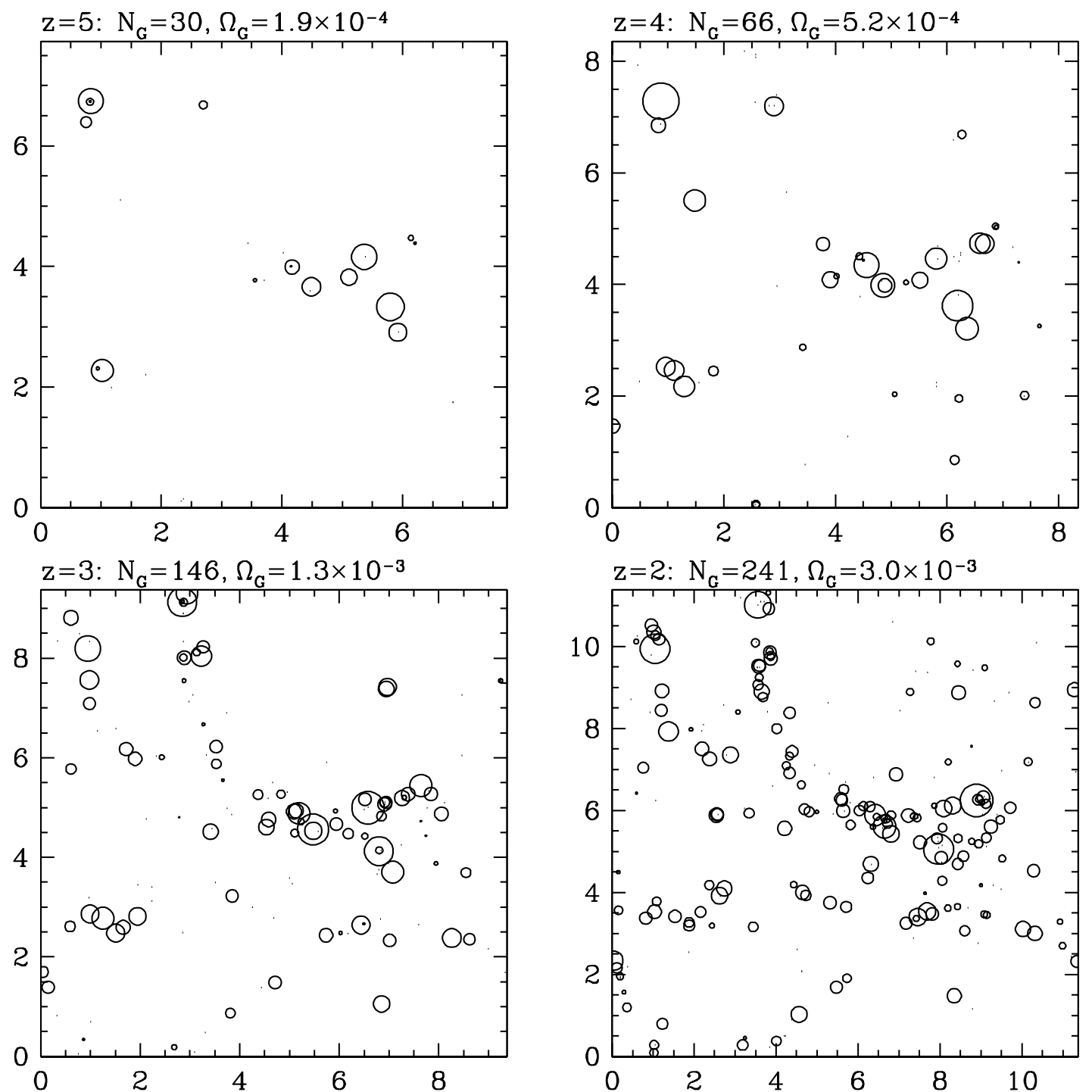

FIG. 7.-Galaxies in the LCDM model at $z=6,5,4,3$, and 2. Each open circle represents a galaxy, with the area of the circle proportional to the galaxy's instantaneous SFR. The label above each panel indicates the redshift, the number of galaxies, and the contribution to the density parameter from material in galaxies (cold gas + stars). Positions are marked in arcminutes; the box size is 11.111 comoving $h^{-1} \mathrm{Mpc}$ at all redshifts. 

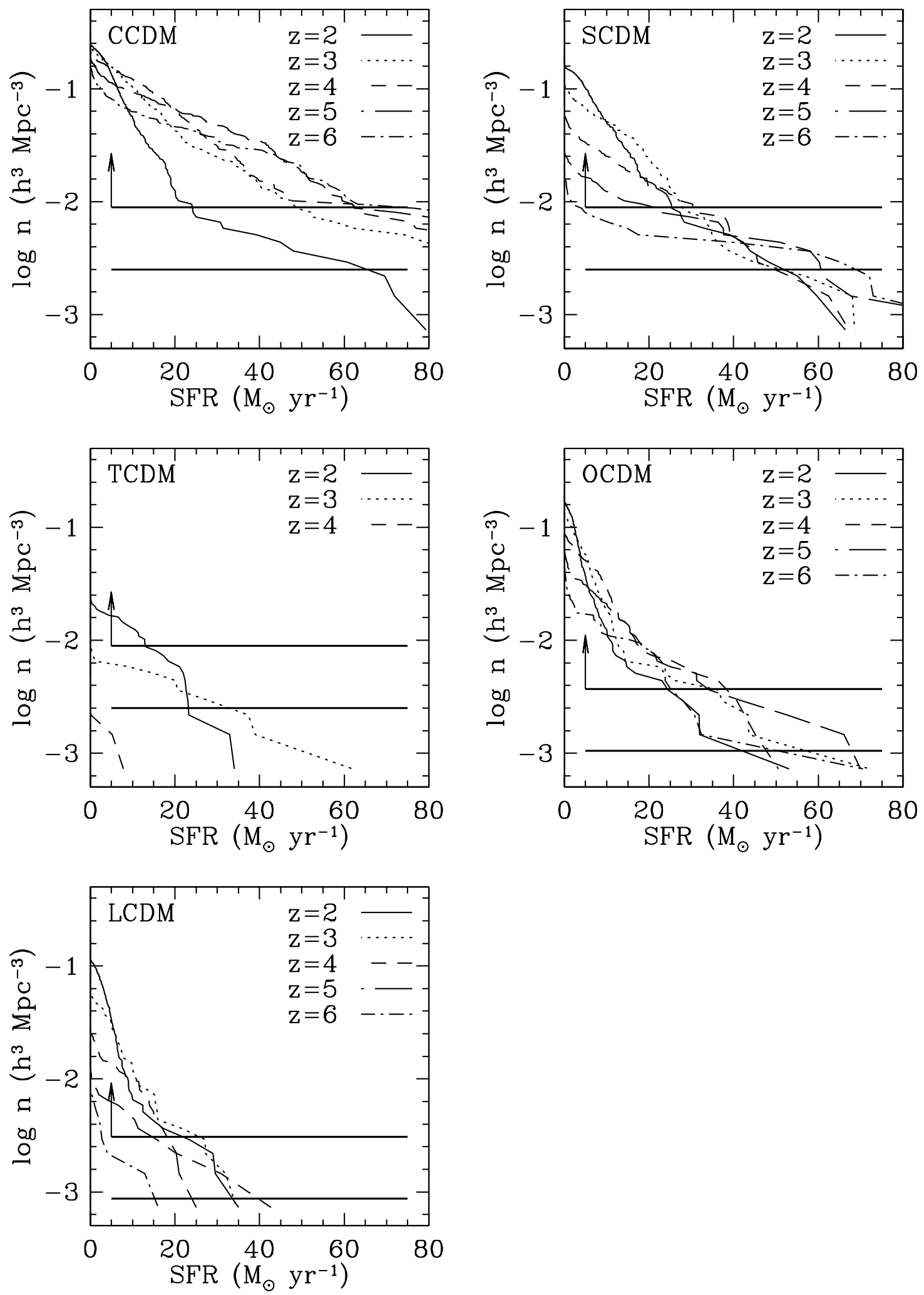

FIG. 8. - Cumulative distribution function of simulated galaxies in terms of instantaneous SFR. Each panel represents a different cosmological model, as indicated. Horizontal lines show the approximate comoving space density of spectroscopically confirmed LBGs at $z=3$ in the samples of Steidel et al. (1996; lower solid lines) and Lowenthal et al. (1997; upper solid lines, with arrow indicating the possible effects of incompleteness in the spectroscopy). If these magnitude-limited samples select the objects with the highest SFRs, then the intersections of the $z=3$ (dotted lines) curves with these horizontal lines indicate the predicted SFRs for objects at the limits of these samples. 
TABLE 2

SFR ANd VOLUme CONVERSIONS

\begin{tabular}{|c|c|c|c|c|c|c|c|c|c|c|c|c|}
\hline \multirow[b]{2}{*}{$\Omega_{m}$} & \multirow[b]{2}{*}{$\Omega_{\Lambda}$} & \multirow[b]{2}{*}{$h$} & \multicolumn{2}{|c|}{$z=6$} & \multicolumn{2}{|c|}{$z=5$} & \multicolumn{2}{|c|}{$z=4$} & \multicolumn{2}{|c|}{$z=3$} & \multicolumn{2}{|c|}{$z=2$} \\
\hline & & & $m_{10}$ & $C_{V}$ & $m_{10}$ & $C_{V}$ & $m_{10}$ & $C_{V}$ & $m_{10}$ & $C_{V}$ & $m_{10}$ & $C_{V}$ \\
\hline $1.0 \ldots \ldots \ldots$ & 0.0 & 0.50 & 25.58 & 191 & 25.30 & 217 & 24.96 & 249 & 24.50 & 285 & 23.82 & 314 \\
\hline $0.4 \ldots \ldots \ldots$ & 0.0 & 0.65 & 25.90 & 621 & 25.55 & 654 & 25.12 & 680 & 24.55 & 684 & 23.73 & 631 \\
\hline $0.4 \ldots \ldots \ldots$ & 0.6 & 0.65 & 25.74 & 590 & 25.45 & 664 & 25.08 & 746 & 24.59 & 824 & 23.86 & 847 \\
\hline
\end{tabular}

NotE. $-m_{10}$ is the $\mathrm{AB}$ magnitude at observed wavelength $\lambda_{\text {obs }}=1500 \times(1+z) \AA$ for an object with unobscured $\mathrm{SFR}=10 M_{\odot} \mathrm{yr}^{-1}$, and multiplication by $C_{V}$ converts space densities from $h^{3} \mathrm{Mpc}^{-3}$ to number $\operatorname{arcmin}^{-2}$ per unit redshift.

tude less than $25.45-2.5 \times \log (20 / 10)=24.70$ at $\lambda_{\text {obs }}=9000 \AA$. The conversions in Table 2 can be calculated using the formulae in Hogg (1999) together with the definition $m_{\mathrm{AB}}=-2.5 \log f_{\nu}-48.60$. Note, however, that these magnitudes assume no dust extinction, while the UV continuum slopes of observed Lyman break galaxies imply typical UV extinctions of 0.5-2.5 mag (Adelberger \& Steidel 2000).

The horizontal lines in Figure 8 mark the comoving space densities of spectroscopically confirmed objects in the LBG samples of Steidel et al. (1996) and Lowenthal et al. (1997), which have a mean redshift $z \approx 3$. Assuming that these surveys pick out the galaxies with the highest star formation rates, the intersections of the dotted $(z=3)$ simulation curves with these horizontal lines yield the predicted star formation rates for galaxies near the sample magnitude limits. The Steidel et al. (1996) magnitude limit of $\mathscr{R} \approx 25.5$ corresponds to a star formation rate of approximately $4 M_{\odot}$ $\mathrm{yr}^{-1}$ with the assumptions described above (see Table 2), but 1-2.5 mag of dust extinction would increase the implied SFR by factors of 2.5-10. The CCDM simulation predicts a star formation rate of $\sim 90 M_{\odot} \mathrm{yr}^{-1}$ for objects with this space density, which is clearly too high unless the true dust extinction is much larger than the Adelberger \& Steidel (2000) estimate. The other simulations predict star formation rates of $\sim 30-50 M_{\odot} \mathrm{yr}^{-1}$, which is consistent with the Steidel et al. (1996) results if the typical extinction is $\sim 2-2.5$ mag. The Lowenthal et al. (1997) survey of the HDF probes 1 mag deeper than the Steidel et al. (1996) sample and thus a factor of 2.5 lower in SFR. The comoving space density of the Lowenthal et al. (1997) galaxies is higher by a factor of 3.5, and since Lowenthal et al. (1997) only observed $\sim \frac{2}{3}$ of their Lyman break candidates (with a $44 \%$ success rate), the true space density at this magnitude limit could be higher by a factor of 1.5-3, as indicated by the arrow in Figure 8. The predicted star formation rates in the CCDM simulation again appear too high, while the predictions of the SCDM, OCDM, and LCDM models appear roughly compatible with the Lowenthal et al. (1997) results if the dust extinction correction is fairly large and the true space density is $2-3$ times the lower limit. The TCDM model predicts very low star formation rates at the Lowenthal et al. (1997) space density. The numerical prediction is clearly ruled out by the data, although this failure of the TCDM model should still be viewed with some caution until it is confirmed at higher numerical resolution. We also note that the small size of the simulation volumes leads to significant uncertainties in the predicted star formation rates at these low comoving densities: the Steidel et al. (1996) space density corresponds to $3.5,1.5$, and 1.2 galaxies in the $\left(11.111 h^{-1} \mathrm{Mpc}\right)^{3}$ simula- tion volume for the critical density, open, and flat- $\Lambda$ models, respectively.

Figure 9 presents a more detailed comparison between numerical predictions and observational data at $z=3$. The upper panel shows the differential distribution of star formation rates. The LCDM result is shown as a solid histogram, but to preserve visual clarity we show distributions for other models as connected lines. Here we omit galaxies with baryonic mass $M_{b}<60 m_{\mathrm{SPH}}$ because limited numerical resolution would cause us to underestimate their star formation rates. The distributions therefore decline at low SFR because of the absence of low-mass galaxies.

In the lower panel of Figure 9 we convert the predictions to observable units using the conversions in Table 2. Points with $1 \sigma$ statistical error bars show the luminosity function of Lyman break galaxies at $z \approx 3$ with (squares) and without (circles) correction for dust extinction, from Adelberger \& Steidel (2000; based on data from Steidel et al. 1999). As Adelberger \& Steidel (2000) emphasize, the extinction-corrected points are quite uncertain; the corrections assume that the correlation between UV continuum slope and extinction observed in local starburst galaxies (Meurer, Heckman, \& Calzetti 1999) also holds at $z=3$, and the points at faint magnitudes rely on an extrapolation of the luminosity function. However, these extinction corrections are probably the best that can be made with current data, and they yield plausible consistency between the population of UV-selected Lyman break galaxies and constraints on dust emission from submillimeter counts and the infrared background (Adelberger \& Steidel 2000).

Given the theoretical and observational uncertainties (which include numerical limitations, IMFs, the value of $\Omega_{b}$, extinction corrections, incompleteness, and $H_{0}$ ), we do not wish to draw strong conclusions from Figure 9. The CCDM model appears to predict excessively luminous galaxies, as expected from the discrepancies already noted, and this discrepancy would be more severe if the simulations had used the Burles \& Tytler $(1997,1998)$ estimate of $\Omega_{b}$ instead of the Walker et al. (1991) estimate. (Gardner et al. $2001 \mathrm{~b}$ find that the SFR scales approximately as $\Omega_{b}^{1.5}$.) The galaxy population in the TCDM model is probably too faint, unless the true extinction corrections are surprisingly small or the numerical resolution effects are more severe than we think. However, a higher $\Omega_{b}$ would improve the performance of this model. The other three models appear roughly compatible with the data. The limited dynamic range of the resolved galaxy populations in the simulations prevents a detailed comparison to the shape of the observed luminosity function. In future work, we will combine simu- 

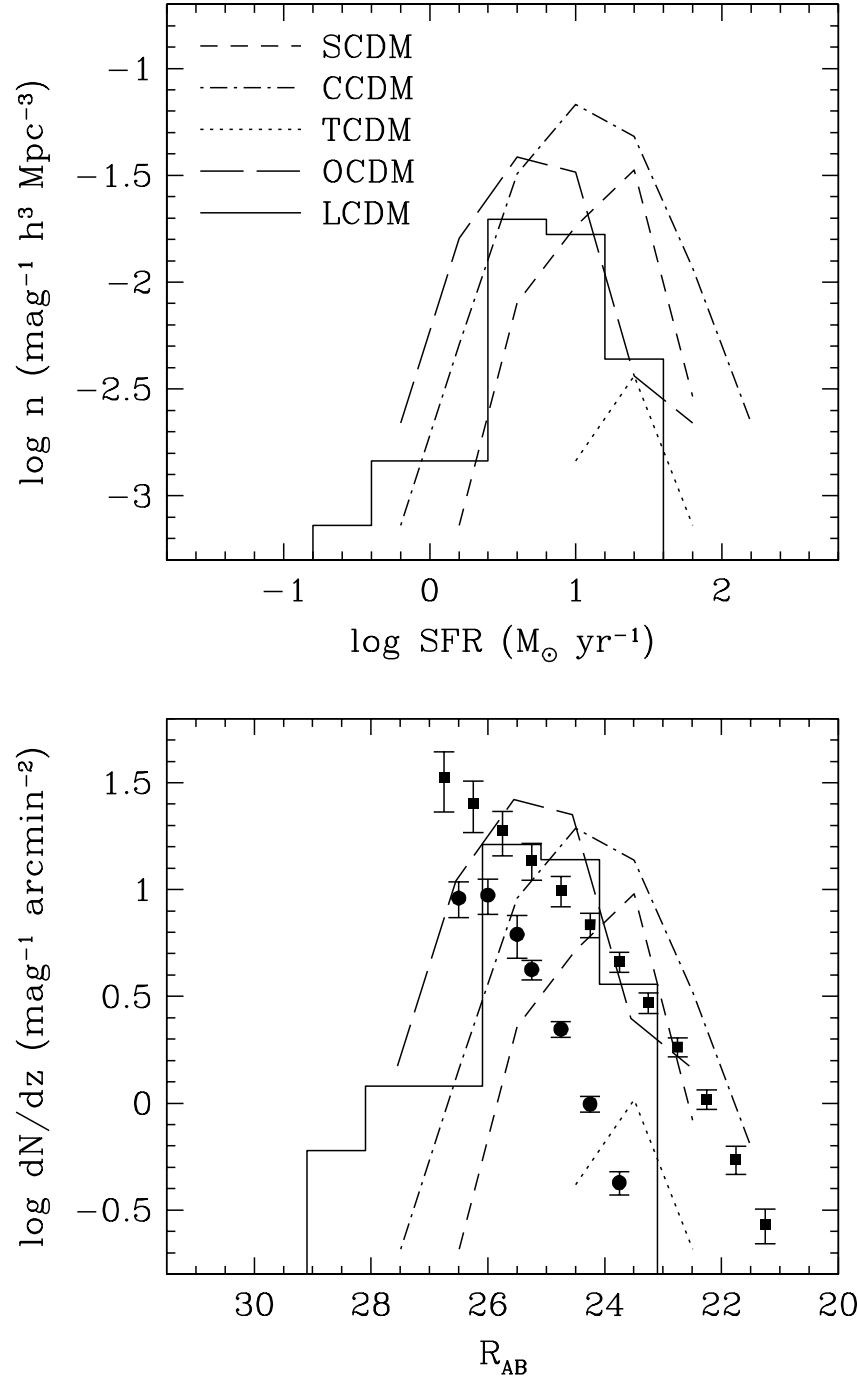

FIG. 9.-Top: Differential distributions of instantaneous SFRs at $z=3$ in the five cosmological models. For visual clarity, only the LCDM result is shown as a histogram, but all distributions are computed as histograms in 1 mag ( 0.4 dex) bins. Only galaxies with baryonic mass $M>60 m_{\mathrm{SPH}}$ are included. Bottom: Same as top, but SFRs have been translated to corresponding $R_{\mathrm{AB}}$ magnitudes as described in the text, and densities have been converted to directly observable units of number $\mathrm{arcmin}^{-2}$ per unit redshift. Points show the luminosity function of LBGs at $z \sim 3$ estimated by Adelberger \& Steidel (2000, Fig. 11c) with (squares) and without (circles) correction for dust extinction.

lations of the LCDM model with different resolutions and box sizes to represent the galaxy population over a wider mass range.

Figure 10 shows the globally averaged density of star formation as a function of redshift, a representation of the cosmic star formation history made famous by Madau et al. (1996). The simulation results accord with the impressions from Figure 8. In particular, comparison of Figure 10 to Figure 1 shows that the cosmic star formation history depends strongly on the amplitude of mass fluctuations. The high-amplitude, CCDM model predicts a high-amplitude SFR curve that peaks at high redshifts. The globally averaged SFR in this model declines slowly at $z<4$. In the other models, the SFR appears to be reaching a plateau at $z \sim 2$, when the simulations stop. The low-amplitude TCDM model predicts the lowest SFR, especially at high

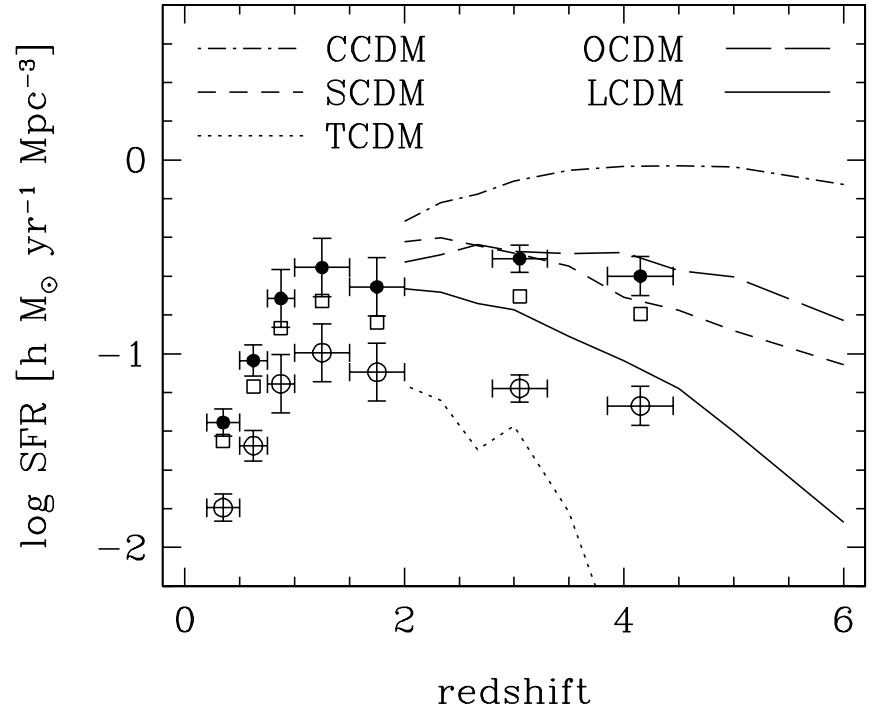

FIG. 10.-Average comoving density of star formation in the five cosmological models, from $z=6$ to $z=2$. Observational estimates with ( filled circles) and without (open circles) corrections for dust extinction are taken from Steidel et al. (1999), based on their own data $(z>3)$ and the data of Lilly et al. (1996; $z<1)$ and Connolly et al. (1997; $1<z<2)$; they are computed for $\Omega_{m}=1, \Omega_{\Lambda}=0$. Open squares show the corresponding extinction-corrected estimates for $\Omega_{m}=0.4, \Omega_{\Lambda}=0.6$. Because observed luminosities scale as $h^{-2}$ and observed space densities as $h^{3}$, we plot the star formation density in the units of $h M_{\odot} \mathrm{yr}^{-1} \mathrm{Mpc}^{-3}$ for which the observational values are independent of the choice of $h$.

redshift. Data points in Figure 10 are taken from the analysis of Steidel et al. (1999), based on their own data and the data of Lilly et al. (1996) and Connolly et al. (1997). At each redshift, Steidel et al. (1999) estimate the global SFR by integrating the UV continuum luminosity function for galaxies with $L>0.1 L_{*}$, so the points do not include the contribution of the faintest galaxies (which are often below the survey magnitude limits). The open circles show estimates with no extinction correction, while the filled circles incorporate extinction corrections of 0.44 mag at $z<2$ and 0.67 mag at $z>2$. Open squares show the extinction-corrected estimates converted to the cosmological parameters of our LCDM model; points for the OCDM model parameters would lie between the open squares and filled circles.

Unfortunately, the global SFR is a difficult quantity to predict robustly from numerical simulations with a limited dynamic range, since they miss the contribution from galaxies below the resolution limit and underestimate the contribution from rare, massive galaxies, which are unlikely to form in a small simulation volume. Figure $2 a$ of Weinberg et al. (1999) illustrates these resolution and box size effects using several simulations of an LCDM model (one with a higher baryon density). Because of these missing contributions, one should regard the curves in Figure 10 as lower limits to the true predictions of these models. The theoretical and observational uncertainties make it difficult to draw strong conclusions from Figure 10. The CCDM model appears to predict too much star formation. The SCDM and OCDM predictions agree fairly well with the extinction-corrected estimates of Steidel et al. (1999), but contributions from lower mass galaxies or an increase in $\Omega_{b}$ would make this agreement worse. The LCDM predictions are somewhat low, but they might plausibly be boosted toward 
reasonable agreement with higher resolution and/or higher $\Omega_{b}$. The TCDM predictions are far below the observational estimates. Of course, most of the action in the observational data takes place at $z<2$, after these simulations stop. Figure $2 b$ of Weinberg et al. (1999) shows preliminary results from simulations of a similar set of models (from Davé et al. 1999), extended to $z=0$. The global SFR declines in all of the models at $z<1$, although not as sharply as the data points in Figure 10.

In Table 3 we list a more robust prediction of the simulations, the contribution to the globally averaged SFR from galaxies above our estimated resolution limit, those with $M_{b}>60 m_{\mathrm{SPH}}$. We also list the number density $n_{\mathrm{res}}$ of such galaxies at each redshift. To the extent that galaxy absolute magnitude correlates with baryonic mass, the corresponding observational quantity could be computed from a galaxy survey by choosing a limiting magnitude at which the galaxy number density is $n_{\text {res }}$ and summing the contribution to the global SFR from galaxies above this magnitude limit.

\section{DISCUSSION}

Our main result is that inflationary CDM models, combined with straightforward assumptions about galactic scale star formation, predict a substantial population of starforming galaxies at $z \geq 2$. The stellar masses and star formation rates of these high-redshift systems are sensitive to the amplitude of the underlying mass power spectrum (e.g., compare Figs. 1 and 10); the cosmological model influences the high- $z$ galaxy population mainly through determining the amplitude of fluctuations on galactic mass scales at the redshifts in question. The results of the LCDM, OCDM, and SCDM simulations appear roughly consistent with the observed properties of Lyman break galaxies, given the theoretical and observational uncertainties. The low-amplitude TCDM model predicts an anemic LBG population that is probably inconsistent with current observations, although this conclusion may be sensitive to our finite numerical resolution and our adopted value of $\Omega_{b}$. The high-amplitude CCDM model appears to predict too much high-redshift star formation, by a significant factor.

The Ly $\alpha$ forest offers a more direct probe of the amplitude of mass fluctuations at high redshift (Croft et al. 1998). Recent observational analyses (Croft et al. 1999, 2001; McDonald et al. 2000) imply that the matter power spectrum at $z \approx 2-3$ is similar to that in our LCDM, OCDM, and SCDM models but incompatible with the CCDM or TCDM models. It is reassuring that the models supported by the Ly $\alpha$ forest data appear to be the most compatible with the star formation properties of observed LBGs, although an increase in $\Omega_{b}$ to the values supported by recent D/H studies (Burles \& Tytler 1997, 1998) and other Ly $\alpha$ forest analyses (Rauch et al. 1997; Weinberg et al. 1997b) might spoil this agreement to some extent. We examine the influence of $\Omega_{b}$ on the high-redshift galaxy population elsewhere (Gardner et al. 2001b), finding that galaxy star formation rates in the SCDM model scale roughly as $\Omega_{b}^{1.5}$. In KHW99, we showed that the clustering of high-redshift galaxies in these simulations is consistent with observed LBG clustering (Adelberger et al. 1998; Giavalisco et al. 1998) and that the clustering is insensitive to the cosmological model because galaxies form at the same "biased" locations of the dark matter distribution in all five simulations.

In Gardner et al. (2001a), we examine the predictions of these simulations for damped $\operatorname{Ly} \alpha$ absorption, which is the other main observational probe of the high-redshift galaxy population. The galaxies resolved in these simulations account for only a fraction of the observed damped Ly $\alpha$ absorption at $z \approx 3$, ranging from $\sim 3 \%$ in TCDM to $\sim 30 \%$ in LCDM, SCDM, and CCDM to $50 \%$ in OCDM. Since the simulations already go to higher space densities than existing spectroscopic samples of LBGs, our results imply that these LBG samples are not yet deep enough to include the galaxies responsible for most damped Ly $\alpha$ absorption. Haehnelt, Steinmetz, \& Rauch (2000) reach a similar conclusion using analytic arguments. By extrapolating the simulation results with the aid of the Jenkins et al. (2001) halo mass function, Gardner et al. (2001a) conclude that absorption in lower mass systems is sufficient to account for observed damped Ly $\alpha$ absorption in any of these cosmological models, with the possible exception of TCDM. However, the uncertainties in the extrapolation are large, and definitive examination of the compatibility between LBG and damped Ly $\alpha$ constraints will require higher resolution simulations.

Submillimeter observations provide important additional constraints on the evolution of star-forming galaxies, since much of the energy emitted by young stars may be absorbed by dust and reradiated in the far-IR. In a recent paper (Fardal et al. 2002), we use larger volume simulations to investigate the submillimeter galaxy population in the LCDM model (with higher $\Omega_{b}$ ). With plausible choices of the dust spectral energy distribution, the simulations can reproduce the observed submillimeter counts and source redshift distribution, but the uncertainties in the observational data and in physical parameters like the dust temperature are

TABLE 3

Comoving Star Formation Density of Resolved Galaxies

\begin{tabular}{|c|c|c|c|c|c|c|c|c|c|c|}
\hline \multirow[b]{2}{*}{ Model } & \multicolumn{2}{|c|}{$z=6$} & \multicolumn{2}{|c|}{$z=5$} & \multicolumn{2}{|c|}{$z=4$} & \multicolumn{2}{|c|}{$z=3$} & \multicolumn{2}{|c|}{$z=2$} \\
\hline & $n_{\text {res }}$ & SFR & $n_{\text {res }}$ & SFR & $n_{\mathrm{res}}$ & SFR & $n_{\text {res }}$ & SFR & $n_{\text {res }}$ & SFR \\
\hline CCDM ........ & 0.067 & 2.733 & 0.099 & 3.386 & 0.138 & 3.380 & 0.168 & 2.909 & 0.195 & 1.748 \\
\hline $\operatorname{SCDM} \ldots \ldots \ldots$ & 0.008 & 0.341 & 0.017 & 0.515 & 0.031 & 0.708 & 0.063 & 1.204 & 0.122 & 1.411 \\
\hline TCDM......... & 0.000 & 0.000 & 0.000 & 0.000 & 0.001 & 0.010 & 0.006 & 0.168 & 0.017 & 0.268 \\
\hline OCDM ........ & 0.017 & 0.326 & 0.035 & 0.554 & 0.061 & 0.732 & 0.095 & 0.739 & 0.137 & 0.658 \\
\hline 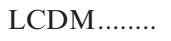 & 0.002 & 0.023 & 0.007 & 0.091 & 0.017 & 0.204 & 0.044 & 0.380 & 0.098 & 0.490 \\
\hline
\end{tabular}

NoTE.-At each redshift, $n_{\text {res }}$ is the comoving number density of simulated galaxies with baryonic mass $M_{b}>60 m_{\mathrm{SPH}}$, in $h^{3} \mathrm{Mpc}^{-3}$, and SFR is the contribution of these galaxies to the comoving star formation density, in $M_{\odot} \mathrm{yr}^{-1} h^{3} \mathrm{Mpc}^{-3}$. 
large enough that this is not a strong test of the model. The infrared background (Hauser \& Dwek 2001) provides another useful constraint on the integrated star formation history, which we will investigate in future work.

Semianalytic methods, sometimes combined with $N$-body simulations of the dark matter distribution, are the main alternative to hydrodynamic simulations for theoretical investigation of high-redshift galaxy formation. Using these methods, several groups have found that CDM models like the ones studied here can reproduce the numbers, luminosities, colors, and clustering properties of observed LBGs (e.g., Baugh et al. 1998; Governato et al. 1998; Kauffman et al. 1999; Somerville et al. 2001). The semianalytic papers have led to three rather different suggestions about the nature of Lyman break galaxies. In the first view, observed LBGs are the most massive galactic systems present at high redshift, forming stars at a fairly steady rate (Baugh et al. 1998). In the second view, interactions play a crucial role in triggering bursts of star formation, and many LBGs are low-mass systems boosted temporarily, and briefly, to prominence (Kolatt et al. 1999; Somerville et al. 2001). A third, intermediate case is one in which LBGs are massive galaxies experiencing bursts of star formation stimulated by minor or major mergers (Somerville et al. 2001). This variety of views is mirrored to some extent in the observational literature on LBGs (e.g., compare Steidel et al. 1996 with Lowenthal et al. 1997, Trager et al. 1997, or Sawicki \& Yee 1998).

Our simulations suggest a picture that is intermediate between the extremes of this debate but closest to the first point of view. Star formation in the simulated galaxies is steady on timescales of $200 \mathrm{Myr}$ (Fig. 3), and the instantaneous star formation rate is fairly well correlated with stellar mass (Fig. 4). However, there is enough scatter in galaxy star formation rates that a sample of galaxies selected above an SFR threshold includes objects with a substantial range of stellar masses (Fig. 4), and these may reside in halos with a wide range of circular velocities (Fig. 6). The simulations automatically include interactions and mergers, but they do not resolve the existence of the low-mass systems envisioned to play an important role in the extreme version of the collision-induced starburst model.

The properties of the simulated LBG population depend on the cosmological initial conditions and on the basic physics of gravity and gas dynamics, but they also depend on our adopted prescription for galactic scale star formation. The crucial features of this prescription are (1) that the local star formation timescale decreases with increasing gas density, as implied by studies of local galaxies (Kennicutt 1998) and (2) that supernova feedback energy is deposited mainly in the dense interstellar medium, where it is usually radiated away before it has a large dynamical impact. Since we do not require any external triggers for star formation, an isolated galaxy that steadily accretes cold gas will convert that gas into stars, albeit over many orbital times. Interactions and mergers can enhance star formation by driving gas to higher density, but galaxies do not build up large reservoirs of dense gas that wait to be ignited. Limited resolution may reduce the influence of interactions and mergers in these simulations, since they do not resolve low-mass satellites and do not resolve the nuclear star formation that is prominent in high-resolution simulations of starbursts induced by minor (Mihos \& Hernquist 1994a, 1996) or major (Mihos \& Hernquist 1994b; Hernquist \& Mihos
1995) mergers. A more efficient feedback mechanism could also lead to more episodic star formation histories by producing cycles of starbursts followed by suppressed accretion and cooling. Since we have not investigated scenarios in which interactions or feedback play a larger role, we cannot draw conclusions about their viability. However, our results suggest that the straightforward treatment of star formation described in $\S 2.1$ is sufficient to explain at least the basic properties of the observed LBG population within the CDM cosmological framework.

Our model of the LBG population is qualitatively consistent with the inferences from recent population synthesis studies of the infrared (rest-frame optical) properties of LBGs (Papovich, Dickinson, \& Ferguson 2001; Pettini et al. 2001; Shapley et al. 2001). While some of the galaxies in these studies appear to be undergoing short bursts of star formation, the majority appear to be converting gas into stars fairly steadily on timescales of a few hundred Myr, consistent with our Figure 3. At the typical space density of the Steidel et al. (1996) and Shapley et al. (2001) samples, corresponding to the top few galaxies in our simulation volume, inferred star formation rates span a wide range with typical values of $\sim 30-100 M_{\odot} \mathrm{yr}^{-1}$, and inferred stellar masses are $\sim 10^{10} M_{\odot}$, in reasonable agreement with our Figure 4. Papovich et al. (2001) also find a correlation between UV luminosity and stellar mass in their HDF sample, as one would expect from our predicted correlation between SFR and $M_{*}$. However, Shapley et al. (2001) find little correlation of these quantities in their sample, possibly because at their higher luminosity threshold the impact of increasing SFR is countered by increasing extinction. The one key feature of LBGs that our simulations do not predict is the presence of large-scale outflows (Franx et al. 1997; Pettini et al. 1998, 2000, 2001), which appear to be nearly ubiquitous in these systems. Because feedback energy is radiated away quickly in the dense interstellar medium of our simulated galaxies, they do not drive galactic scale winds. Simulations that incorporate a multiphase ISM (e.g., Yepes et al. 1997; Springel 2000) might produce outflows; it is unclear whether these would alter star formation properties in a way that would spoil the otherwise good agreement with the observations.

The properties of LBGs in our LCDM simulation appear fairly consistent with those in the LCDM hydrodynamic simulation analyzed by Nagamine et al. (2001a, 2001b) and Nagamine (2002). We have focused our analyses on somewhat different issues, precluding a detailed comparison of results, but both studies agree that the LCDM model predicts typical star formation rates $\sim 10-100 M_{\odot} \mathrm{yr}^{-1}$ and stellar masses $\sim 10^{10} M_{\odot}$ for LBGs with the space density of current spectroscopic samples, and both find that star formation in the simulated galaxies is fairly steady on timescales of 200 Myr. Nagamine et al. use an Eulerian grid simulation that has much higher mass resolution (baryon mass of $4.8 \times 10^{5} M_{\odot}$ per cell vs. our $6.7 \times 10^{7} M_{\odot}$ per SPH particle) but much lower force resolution (comoving cell size of $32.6 h^{-1} \mathrm{kpc}$ vs. our comoving Plummer softening of $3.5 h^{-1} \mathrm{kpc}$ ). The formulae for star formation rate are similar in the two calculations, but the overdensity threshold for star formation is only 5.5 in the Nagamine et al. simulation, while our physical threshold of $n_{\mathrm{H}}=0.1$ atoms $\mathrm{cm}^{-3}$ corresponds to an overdensity of $1.5 \times 10^{4}$ at $z=3$. Given the major differences in the numerical approach and the mass and force resolution of the 
calculations, the level of consistency between our results is encouraging.

The clearest prediction of our simulations is that the Lyman break galaxies studied by Steidel et al. $(1996,1999)$ and Lowenthal et al. (1997) represent the tip of an iceberg. The cumulative distribution curves in Figure 8 should be taken as lower limits to the predicted galaxy number densities, especially at high redshifts, since limited resolution causes these simulations to underestimate the star formation rates in low-mass systems, or to miss the systems entirely. Nonetheless, the curves for, e.g., the LCDM model show that there should be large numbers of $z=3$ galaxies below the magnitude limits of current LBG samples, and significant numbers of galaxies with SFR $\geq 10 M_{\odot} \mathrm{yr}^{-1}$ even at $z \geq 5$. Recent searches have already yielded a number of spectroscopically confirmed galaxies at $z=5-7$ (Spinrad et al. 1998; Weymann et al. 1998; Chen, Lanzetta, \& Pascarelle 1999; Hu, Cowie, \& McMahon 1999), and analyses of deep $H S T$ /NICMOS images show candidate objects to redshifts $z \geq 10$ (Yahata et al. 2000; Dickinson 2000). Systematic characterization of this faint galaxy population will be challenging, so it will be some time before simulations and data can be compared quantitatively in the very high redshift regime. But the existence of a significant population of starforming galaxies at $z>5$ is a natural prediction of the CDM scenario.

This paper presents a first attempt to characterize the star formation properties of high-redshift galaxies using hydrodynamic simulations, but there is much room for progress with future simulations. The emerging consensus on cosmological parameters, if it survives the tightening of observational constraints, makes the task easier by focusing effort on a preferred background model. Within such a framework, simulations with different box sizes and resolutions can be combined to model the galaxy population over a wider dynamic range of mass and redshift, improving the comparison to the observed luminosity function (as in Fig. 9) and global star formation history (as in Fig. 10). Weinberg et al. (1999) take a first step along this path, using multiple simulations of the LCDM model (with higher $\Omega_{b}$ ) to predict cumulative SFR distributions from $z=0.5$ to $z=10$. Since the present simulations resolve galaxies far below the limits of current LBG spectroscopic samples, simulations of larger volumes at lower resolution will improve the comparison between predicted and observed LBG clustering. Higher resolution simulations, on the other hand, can probe the connection between LBGs and damped Ly $\alpha$ systems and test the robustness of some of the trends found in $\S 3$. They can also provide predictions of the structural properties of high-redshift galaxies, such as size and morphology, although these may be best investigated with simulations that zoom in to follow the formation of individual objects (e.g., Haehnelt, Steinmetz, \& Rauch 1998; Contardo, Steinmetz, \& Fritze-von Alvensleben 1998). In the long run, we also hope to examine different formulations of star formation and feedback, to determine what descriptions of these physical processes match the observed properties of galaxies and the intergalactic medium over the full range of accessible redshifts.

Where are the Lyman break galaxies today? Because our present simulations stop at $z=2$, we will save a detailed examination of this question for a future paper on the assembly history of galaxies, using simulations (like those of Davé et al. 1999) that continue to $z=0$. A first look at these simulations suggests that there is no simple, one-sentence answer. Between $z=3$ and $z=0$, galaxies accrete fresh material and merge with each other, and many new galaxies form that had no $z=3$ progenitors at all (at least above the numerical resolution limits). The particles that lie in galaxies at $z=3$ are distributed at $z=0$ among galaxies with a wide range of environments and masses, although the most massive $z=0$ galaxies always contain some of these particles and the least massive often do not. Any link between LBGs and present-day ellipticals, bulges, halos, or clusters, can at best capture a general trend, one that is likely to be violated nearly as often as it is obeyed. Fortunately, cosmological simulations are an ideal tool for characterizing the full range of possible histories, providing the theoretical thread that can tie snapshots of the galaxy population at different redshifts into a coherent picture of galaxy formation and evolution.

We thank Kurt Adelberger and Chuck Steidel for helpful discussions and for providing the data plotted in Figures 9 and 10. We also thank James Lowenthal for helpful discussions. This work was supported by NASA Astrophysical Theory Grants NAG5-3922, NAG5-3820, and NAG53111, by NASA Long-Term Space Astrophysics Grant NAG5-3525, and by the NSF under grants ASC93-18185, ACI96-19019, and AST-9802568. The simulations were performed at the San Diego Supercomputer Center.

\section{REFERENCES}

Adelberger, K. L., \& Steidel, C. C. 2000, ApJ, 544, 218

Adelberger, K. L., Steidel, C. C., Giavalisco, M., Dickinson, M., Pettini, M., \& Kellogg, M. 1998, ApJ, 505, 18

Bahcall, N. A., Ostriker, J. P., Perlmutter, S., \& Steinhardt, P. J. 1999, Science, 284, 1481

Bardeen, J., Bond, J. R., Kaiser, N., \& Szalay, A. S. 1986, ApJ, 304, 15

Barnes, J. E., \& Hut, P. 1986, Nature, 324, 446

Baugh, C. M., Cole, S., Frenk, C. S., \& Lacey, C. G. 1998, ApJ, 498, 504

Bennett, C., L., et al. 1996, ApJ, 464, L1

Bruzual, A. G., \& Charlot, S. 1993, ApJ, 405, 538

Bunn, E. F., \& White, M. 1997, ApJ, 480, 6

Burles, S., \& Tytler, D. 1997, AJ, 114, 1330

1998, ApJ, 499, 699

Chen, H.-W., Lanzetta, K. M., \& Pascarelle, S. 1999, Nature, 398, 586

Cole, S., Weinberg, D. H., Frenk, C. S., \& Ratra, B. 1997, MNRAS, 289, 37

Connolly, A. J., Szalay, A. S., Dickinson, M., SubbaRao, M. U., \& Brunner, R. J. 1997, ApJ, 486, L11

Contardo, G., Steinmetz, M., \& Fritze-von Alvensleben, U. 1998, ApJ, 507,497
Croft, R. A. C., Weinberg, D. H., Bolte, M., Burles, S., Hernquist, L., Katz, N., Kirman, D., \& Tytler, D. 2001, ApJ, submitted (astro-ph/0012324)

Croft, R. A. C., Weinberg, D. H., Katz, N., \& Hernquist, L. 1997, ApJ, 488,532

488, 1998, ApJ, 495, 44

Croft, R. A. C., Weinberg, D. H., Pettini, M., Katz, N., \& Hernquist, L. 1999, ApJ, 520, 1

Davé, R., Hernquist, L., Katz, N., \& Weinberg, D. H. 1999, ApJ, 511, 521

Dickinson, M. 2000, Philos. Trans. R. Soc. London A, 358, 2001

Efstathiou, G., Bond, J. R., \& White, S. D. M. 1992, MNRAS, 258, 1

Fardal, M. A., Katz, N., Weinberg, D. H., Davé, R., \& Hernquist, L. 2002, ApJ, in press

Franx, M., Illingworth, G. D., Kelson, D. D., van Dokkum, P. G., \& Tran, K. 1997, ApJ, 486, L75

Gardner, J. P., Katz, N., Hernquist \& Weinberg, D. H. 1997, ApJ, 484, 31 2001a, ApJ, 559, 131

2001b, ApJ, submitted (astro-ph/0107293)

Gelb, J. M., \& Bertschinger, E. 1994, ApJ, 436, 467

Giavalisco, M., Steidel, C. C., Adelberger, K. L., Dickinson, M. E., Pettini, M., \& Kellogg, M. 1998, ApJ, 503, 543 
Gingold, R. A., \& Monaghan, J. J. 1977, MNRAS, 181, 375

Gorski, K. M., Banday, A. J., Bennett, C. L., Hinshaw, G., Kogut, A., Smoot, G. F., \& Wright, E. L. 1996, ApJ, 464, L11

Governato, F., Baugh, C. M., Frenk, C. S., Cole, S., Lacey, C. G., Quinn, T. R., \& Stadel, J. 1998, Nature, 392, 359

Haardt, F., \& Madau, P. 1996, ApJ, 461, 20

Haehnelt, M. G., Steinmetz, M., \& Rauch, M. 1998, ApJ, 495, 647 2000, ApJ, 534, 594

Hauser, M. G., \& Dwek, E. 2001, ARA\&A, 39, 249

Hernquist, L., \& Katz, N. 1989, ApJS, 70, 419

Hernquist, L., \& Mihos, J. C. 1995, ApJ, 448, 41

Hogg, D. W. 1999, preprint (astro-ph/9905116)

Hu, E. M., Cowie, L. L., \& McMahon, R. G. 1999, ApJ, 522, L9

Hu, W., \& Sugiyama, N. 1996, ApJ, 471, 542

Jenkins, A., Frenk, C. S., White, S. D. M., Colberg, J. M., Cole, S., Evrard,

A. E. Couchman, H. M. P., \& Yoshida, N. 2001, MNRAS, 321, 372

Katz, N., Hernquist, L., \& Weinberg, D. H. 1999, ApJ, 523, 463 (KHW99)

Katz, N., Weinberg, D. H., \& Hernquist, L. 1996, ApJS, 105, 19 (KWH96)

Kauffmann, G., Colberg, J. M., Diaferio, A., \& White, S. D. M. 1999, MNRAS, 307, 529

Kennicutt, R. C. 1998, ApJ, 498, 541

Kolatt, T. S., et al. 1999, ApJ, 523, L109

Lehnert, M. D., \& Heckman, T. M. 1996, ApJ, 472, 546

Lilly, S. J., LeFevre, O., Hammer, F., \& Crampton, D. 1996, ApJ, 460, L1

Lowenthal, J. D., et al. 1997, ApJ, 481, 673

Lucy, L. 1977, AJ, 82, 1013

Madau, P. 1997, in The Hubble Deep Field, ed. M. Livio, S. M. Fall, \& P. Madau (Cambridge: Cambridge Univ. Press), 200

Madau, P., Ferguson, H. C., Dickinson, M. E., Giavalisco, M., Steidel, C. C., \& Fruchter, A. 1996, MNRAS, 283, 1388

McDonald, P., Miralda-Escudé, J., Rauch, M., Sargent, W. L. W., Barlow,

T. A., Cen, R., \& Ostriker, J. P. 2000, ApJ, 543, 1

Meurer, G. R., Heckman, T. M., \& Calzetti, D. 1999, ApJ, 521, 64

Mihos, J. C., \& Hernquist, L. 1994a, ApJ, 425, L13 $1994 b$, ApJ, 431, L9 1996, ApJ, 464, 641

Miller, G. E., \& Scalo, J. M. 1979, ApJS, 41, 513

Monaghan, J. J. 1992, ARA\&A, 30, 543

Nagamine, K. 2002, ApJ, 564, 73

Nagamine, K., Fukugita, M., Cen, R., \& Ostriker, J. P. 2001a, ApJ, 558, 497

2001b, MNRAS, 327, L10

Papovich, C., Dickinson, M., \& Ferguson, H. C. 2001, ApJ, 559, 620

Peacock, J. A., \& Dodds, S. J. 1994, MNRAS, 267, 1020
Pettini, M., Kellogg, M., Steidel, C. C., Dickinson, M., Adelberger, K. L., \& Giavalisco, M. 1998, ApJ, 508, 539

Pettini, M., Shapley, A. E., Steidel, C. C., Cuby, J., Dickinson, M., Moorwood, A. F. M., Adelberger, K. L., \& Giavalisco, M. 2001, ApJ, 554,981

Pettini, M., Steidel, C. C., Adelberger, K. L., Dickinson, M., \& Giavalisco, M. 2000, ApJ, 528, 96

Ratra, B., Sugiyama, N., Banday, A. J., \& Górski, K. M. 1997, ApJ, 481, 22

Rauch, M., et al. 1997, ApJ, 489, 7

Sawicki, M., \& Yee, H. K. C. 1998, AJ, 115, 1329

Schmidt, M. 1959, ApJ, 129, 243

Seljak, U., \& Zaldarriaga, M. 1996, ApJ, 469, 437

Shapley, A. E., Steidel, C. C., Adelberger, K. L., Dickinson, M., Giavalisco, M., \& Pettini, M. 2001, ApJ, 562, 95

Smoot, G. F., et al. 1992, ApJ, 396, L1

Somerville, R. S., Primack, J. R., \& Faber, S. M. 2001, MNRAS, 320,504

Spinrad, H., Stern, D., Bunker, A., Dey, A., Lanzetta, K., Yahil, A.,

Pascarelle, S., \& Fernandez-Soto, A. 1998, AJ, 116, 2617

Springel, V. 2000, MNRAS, 312, 859

Steidel, C., Adelberger, K., Giavalisco, M., Dickinson, M., \& Pettini, M. 1999, ApJ, 519, 1

Steidel, C. C., Giavalisco, M., Pettini, M., Dickinson, M. E., \& Adelberger, K. L. 1996, ApJ, 462, L17

Trager, S., Faber, S., Dressler, A., \& Oemler, A. 1997, ApJ, 485, 92

Walker, T. P., Steigman, G., Schramm, D. N., Olive, K. A., \& Kang, H. S. 1991, ApJ, 376, 51

Wang, X., Tegmark, M., \& Zaldarriaga, M. 2002, Phys. Rev. D, in press

Weinberg, D. H., Davé, R., Gardner, J. P., Hernquist, L., \& Katz, N. 1999, in ASP Conf. Ser. 191, Photometric Redshifts and High Redshift Galaxies, ed. R. Weymann et al. (San Francisco: ASP), 341

Weinberg, D. H., Hernquist, L., \& Katz, N. 1997a, ApJ, 477, 8

Weinberg, D. H., Miralda-Escudé, J., Hernquist, L., \& Katz, N. 1997b, ApJ, 490, 564

Weymann, R. J., Stern, D., Bunker, A., Spinrad, H., Chaffee, F. H., Thompson, R. I., \& Storrie-Lombardi, L. J. 1998, ApJ, 505, L95

White, S. D. M., Efstathiou, G. P., \& Frenk, C. S. 1993, MNRAS, 262, 1023

Williams, R. E., et al. 1996, AJ, 112, 1335

Yahata, N., Lanzetta, K. M., Chen, H.-W., Ferandez-Soto, A., Pascarelle, S. M., Yahil, A., \& Puetter, R. C. 2000, ApJ, 538, 493

Yepes, G., Kates, R., Khokhlov, A., \& Klypin, A. 1997, MNRAS, 284, 235

Zaldarriaga, M., Seljak, U., \& Bertschinger, E. 1998, ApJ, 494, 491 